\title{
Efficient treatment of stress singularities in poroelastic Wave Based models using special purpose enrichment functions
}

\author{
Elke Deckers*, Bert Van Genechten, Dirk Vandepitte and Wim Desmet \\ K.U.Leuven - Dept. of Mechanical Engineering, Celestijnenlaan 300B - box 2420, 3001 Heverlee (Leuven), \\ Belgium
}

\begin{abstract}
The Finite Element Method is the most commonly used prediction technique to model the harmonic behavior of poroelastic materials. A major disadvantage of this method is its practical frequency limitation in that the computational efforts become prohibitively large at higher frequencies. A recently developed Wave Based Method is an efficient alternative deterministic prediction technique which aims at relaxing this frequency limitation by using exact solutions of the governing equations to approximate the field variables. This paper discusses the application of the Wave Based Method for the particular case that stress singularities are present in corners of the poroelastic domain. Based on an asymptotic analysis, the paper derives a criterion to predict the presence of stress singularities and proposes a suitable set of enrichment functions to extend the conventional set of expansion functions. The beneficial effect of incorporating these functions on the convergence of the Wave Based Method is illustrated by means of a numerical validation study.
\end{abstract}

Key words: poroelastic materials, Biot theory, stress singularities, wave based method, Trefftz approach

\section{Introduction}

Poroelastic media constitute an important class of materials which are commonly used in a.o. passive noise control treatments. Within such a material, the two interpenetrating continua, i.e. the solid phase (or frame) and the fluid phase contained within its pores, exhibit a strong mutual interaction. The most commonly applied mathematical model to describe the dynamic behavior of such materials is the Biot theory [1,2]. Using a set of two frequency-dependent coupled partial differential equations, this constitutive model predicts the existence of three different fluid-frame coupled wave types: a shear wave and two compressional waves.

Currently, the Finite Element Method (FEM) is the most commonly used prediction technique for the analysis of those materials. Even though several alternative formulations exist [3, 4, 5, 6, 7], these calculations are very time-consuming due to the complex and frequency dependent material properties, the high number of unknowns per node and the dense problem discretizations needed

${ }^{*}$ Corresponding author. Address: K.U.Leuven - Dept. of Mechanical Engineering, Celestijnenlaan 300B - box 2420, 3001 Heverlee (Leuven), Belgium. Tel.:+32 163225 94; fax: +32 16322987.

E-mail address: Elke.Deckers@mech.kuleuven.be 
to capture the short wavelengths in the poroelastic response fields at higher frequencies. As a result, the use of the FEM is practically limited to low-frequency applications [8]. The standard FE formulations are improved by using hierarchical finite elements $[9,10]$, but more general trends for the convergence of these schemes for poroelastic materials have to be derived. The embedding of waves as unknowns in standard FE interpolations [11] may also be effective to tackle this type of problems more efficiently.

The Wave Based Method (WBM) [12] is a deterministic method which is based on an indirect Trefftz approach [13]. Instead of discretizing the problem domain into a large number of small elements, the WBM partitions the problem domain into a small number of large, convex subdomains. In each subdomain, the dynamic response variables are approximated using so-called wave functions which are exact solutions of the governing differential equations. In this way, the wave functions may only violate the boundary and continuity conditions. Enforcing the errors on the boundaries and interfaces to zero through a weighted residual formulation yields a small matrix equation which can be solved for the contribution factor of each wave function. In the past similar WB modeling approaches have been successfully applied to uncoupled acoustic [14, 15], uncoupled elastic [16, 17] and fully coupled vibro-acoustic problems $[18,19]$. In recent work $[20,21]$, the authors have proposed a WBM for the solution of the Biot equations. The dynamic field variables are approximated using a superposition of three types of globally defined wave functions, corresponding to the three aforementioned poroelastic wave types. By embedding a priori knowledge in the numerical scheme a significant increase in modeling efficiency is realized and accurate response predictions in an extended frequency range become feasible.

Due to the linearized underlying constitutive model, the poroelastic stress fields may become infinite at points where concentrated loads are applied or at discontinuities along the problem boundary [22]. The latter type of singularities can be expected at the corner points of a polygonal problem domain. When using the FEM, these phenomena do not interfere with the overall prediction accuracy due to the local support of the FE shape functions. In the WB approach, a more profound impact on the predicted response fields is observed due to the global nature of the applied approximation functions. A possible solution to cope with these problems which obviously impact the convergence rate is to extend the set of wave functions using some additional enrichment functions, which accurately represent of the singular behavior of the dynamic fields near the corner point. Similar to the work by Vanmaele et al. [17] on the treatment of corner stress singularities in plate bending problems, this paper derives a generic criterion for the presence of stress singularities in the poroelastic responses based on the internal angle of each corner and the applied boundary conditions. Using an asymptotic response analysis a suitable set of enrichment functions is proposed. The beneficial effect of incorporating these functions in the field variable approximations on the convergence of the WBM is illustrated by means of a numerical validation study.

This paper is organized as follows. Section 2 discusses the mathematical formulation of a general coupled two-dimensional poroelastic-acoustic problem and presents the most commonly applied boundary conditions. Section 3 first describes the problem setting of a general bounded steady-state dynamic problem governed by a system of Helmholtz partial differential equations and addresses the WB modeling approach thereof. Subsequently the WBM is applied to both the analysis of acoustic and poroelastic problems. Section 4 deals with the existence of stress singularities in a poroelastic domain and derives enrichment functions to capture the singular behavior at corner points of a poroelastic domain. The use of the WBM for poroelastic materials is demonstrated in section 5 and it is shown that adding the enrichment functions to the set of wave functions is beneficial for the convergence rate of the method. The paper ends with some concluding remarks and topics for 
future research.

\section{Problem definition}

This section briefly describes the mathematical problem setting used for a general 2D coupled acoustic-poroelastic steady-state problem, as shown in figure 1. A harmonic time dependence $e^{j \omega t}$ is assumed. $j$ is the imaginary unit, $\omega=2 \pi f$ is the circular frequency and $t$ is the time. The total problem domain $\Omega$ can be divided into two subdomains $\Omega_{a}$ and $\Omega_{p e}$ containing respectively air and a poroelastic medium. This section presents the governing equations for both subdomains, reviews the most commonly applied boundary conditions and gives the coupling conditions between the acoustic and the poroelastic domain.

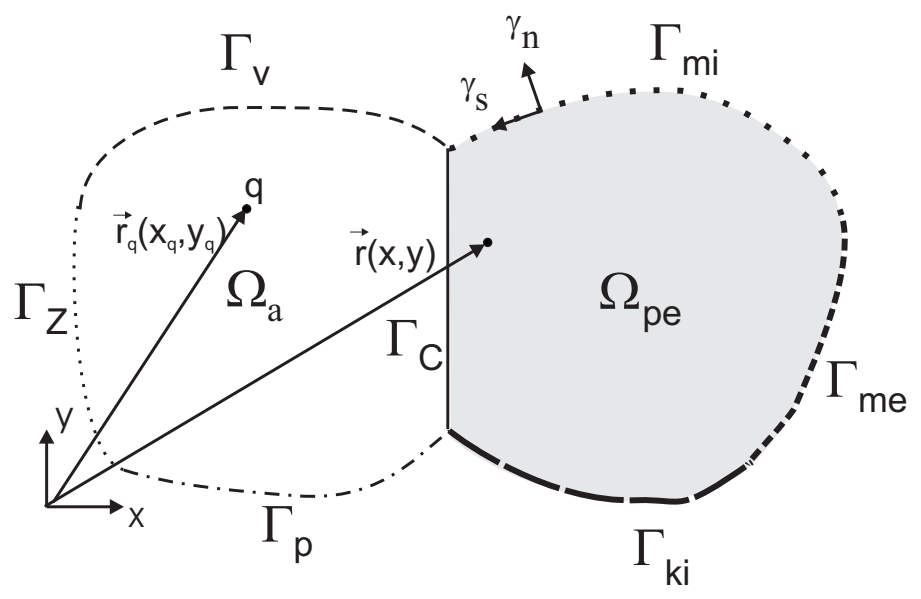

Figure 1: 2D convex poroelastic domain

\subsection{Steady-state interior acoustic problem}

Under the assumption of an inviscid fluid with ambient fluid density $\rho_{f}$ and speed of sound $c$, a linear system and an adiabatic process, the steady-state acoustic pressure $p(\mathbf{r})$ inside the problem domain is governed by the inhomogeneous Helmholtz equation:

$$
\nabla^{2} p(\mathbf{r})+k_{f}^{2} p(\mathbf{r})=-j \rho \omega \delta\left(\mathbf{r}, \mathbf{r}_{\mathbf{q}}\right) q,
$$

In this equation, $\nabla^{2}$ is the Laplacian operator, $k_{f}=\omega / c_{f}$ is the acoustic wave number, $\delta$ is the Dirac delta function and $q$ the amplitude of an acoustic point source at position $\mathbf{r}_{\mathbf{q}}$. Since the Helmholtz equation is a second order differential equation, one boundary condition needs to be specified at each point of the boundary in order to obtain a well-posed problem. The boundary of the considered acoustic subdomain $\Omega_{a}$ is denoted as $\Gamma_{a}=\partial \Omega_{a}$ and consists of two parts, the exterior boundary $\Gamma_{a e}$ and the coupling face with the poroelastic domain $\Gamma_{C}: \Gamma_{a}=\Gamma_{a e} \bigcup \Gamma_{C}$. $\Gamma_{a e}\left(=\Gamma_{v} \bigcup \Gamma_{p} \bigcup \Gamma_{Z}\right)$ represents the part of the boundary $\Gamma_{a}$ at which one of the three following acoustic boundary residuals are defined: 
- acoustic normal velocity boundary conditions:

$$
\mathbf{r} \in \Gamma_{v}: \quad R_{v}(\mathbf{r})=\mathcal{L}_{v}(p(\mathbf{r}))-\bar{v}_{n}=0
$$

with $\bar{v}_{n}$ the prescribed value for the normal acoustic velocity.

- acoustic normal impedance boundary conditions:

$$
\mathbf{r} \in \Gamma_{Z}: \quad R_{Z}(\mathbf{r})=\mathcal{L}_{v}(p(\mathbf{r}))-\frac{p(\mathbf{r})}{\bar{Z}_{n}}=0
$$

with $\bar{Z}_{n}$ the prescribed value for the normal acoustic impedance.

- acoustic pressure boundary conditions:

$$
\mathbf{r} \in \Gamma_{p}: \quad R_{p}(\mathbf{r})=p(\mathbf{r})-\bar{p}=0
$$

with $\bar{p}$ the prescribed value for the acoustic pressure.

The velocity operator is defined as:

$$
\mathcal{L}_{v}=\frac{j}{\rho_{f} \omega} \frac{\partial}{\partial \gamma_{n}}
$$

with $\gamma_{\mathbf{n}}$ the normal direction of the boundary. $\Gamma_{C}$ corresponds to the part of the boundary that coincides with the poroelastic domain. The coupling conditions between an acoustic and a poroelastic subdomain will be explained later.

\subsection{Steady-state interior poroelastic problem}

A poroelastic material consist of two phases, a solid phase $\left(\bullet^{s}\right)$ and a fluid phase $\left(\bullet^{f}\right)$ contained within the pores. The Biot theory $[1,2]$ assumes that the pores are homogeneously distributed in the material and uses an equivalent solid and a compressible fluid continuum description on a macroscopic level. This is justified in the case that the pore dimensions are small as compared to the wavelengths of the different waves which propagate through the medium. The momentum equations may be written as:

$$
\begin{aligned}
& \nabla \cdot \sigma^{\mathbf{s}}(\mathbf{r})=-\omega^{2} \rho_{1} \mathbf{u}^{\mathbf{s}}(\mathbf{r})-\left(\omega^{2} \rho_{a}-j \omega b\right)\left(\mathbf{u}^{\mathbf{s}}(\mathbf{r})-\mathbf{u}^{\mathbf{f}}(\mathbf{r})\right) \\
& \nabla \cdot \sigma^{\mathbf{f}}(\mathbf{r})=-\omega^{2} \rho_{2} \mathbf{u}^{\mathbf{f}}(\mathbf{r})-\left(\omega^{2} \rho_{a}-j \omega b\right)\left(\mathbf{u}^{\mathbf{f}}(\mathbf{r})-\mathbf{u}^{\mathbf{s}}(\mathbf{r})\right)
\end{aligned}
$$

The constitutive relations are:

$$
\begin{aligned}
\sigma^{\mathbf{s}}(\mathbf{r}) & =\left[\left(\lambda+\frac{Q^{2}}{R}\right) e^{s}(\mathbf{r})+Q e^{f}(\mathbf{r})\right] \mathbf{I}+2 N \mathbf{e}^{\mathbf{s}}(\mathbf{r}) \\
\sigma^{\mathbf{f}}(\mathbf{r}) & =\left[Q e^{s}(\mathbf{r})+R e^{f}(\mathbf{r})\right] \mathbf{I}
\end{aligned}
$$

The substitution of the constitutive relations (8)-(9) into the momentum equations (6)-(7) leads to the well-known Biot equations:

$$
\begin{aligned}
N \nabla^{2} \mathbf{u}^{\mathbf{s}}(\mathbf{r})+\nabla\left[\left(\lambda+\frac{Q^{2}}{R}+N\right) e^{s}(\mathbf{r})+Q e^{f}(\mathbf{r})\right] & =-\omega^{2}\left(\rho_{11}^{*} \mathbf{u}^{\mathbf{s}}(\mathbf{r})+\rho_{12}^{*} \mathbf{u}^{\mathbf{f}}(\mathbf{r})\right) \\
\nabla\left[Q e^{s}(\mathbf{r})+R e^{f}(\mathbf{r})\right] & =-\omega^{2}\left(\rho_{12}^{*} \mathbf{u}^{\mathbf{s}}(\mathbf{r})+\rho_{22}^{*} \mathbf{u}^{\mathbf{f}}(\mathbf{r})\right)
\end{aligned}
$$


In these equations, $\mathbf{u}^{\bullet}(\mathbf{r})$ is a displacement vector, $\mathbf{e}^{\bullet}(\mathbf{r})$ the strain vector, $e^{\bullet}(\mathbf{r})$ the longitudinal strain, $\sigma^{\bullet}$ the stress tensor in phase $\bullet$ and $\lambda, Q, R, N, \rho_{a}, \rho_{11}^{*}, \rho_{12}^{*}, \rho_{22}^{*}$ and $b$ are complex valued material properties which depend on the angular frequency. All the parameters used in equations (6)-(10) are defined in Appendix A. For a complete description and interpretation, the reader is referred to literature $[2,9,21]$.

For a poroelastic material, three boundary conditions have to be specified at each point of the boundary in order to have a well-posed problem. The boundary of the subdomain $\Gamma_{p e}=\partial \Omega_{p e}$ consists of an exterior boundary $\Gamma_{p e e}$ and the interface with the acoustic domain $\Gamma_{C} \cdot \Gamma_{p e e}(=$ $\left.\Gamma_{k i} \bigcup \Gamma_{m e} \bigcup \Gamma_{m i}\right)$ represents the part of the boundary $\Gamma_{p e}$ along which one of the three following sets of boundary residuals are defined:

- kinematic boundary conditions, the displacement components are prescribed:

$$
\mathbf{r} \in \Gamma_{k i}:\left\{\begin{array}{l}
R_{u_{n}^{s}}(\mathbf{r})=u_{n}^{s}(\mathbf{r})-\bar{u}_{n}^{s}(\mathbf{r})=0 \\
R_{u_{s}^{s}}(\mathbf{r})=u_{s}^{s}(\mathbf{r})-\bar{u}_{s}^{s}(\mathbf{r})=0 \\
R_{u_{n}^{f}}(\mathbf{r})=u_{n}^{f}(\mathbf{r})-\bar{u}_{n}^{f}(\mathbf{r})=0
\end{array}\right.
$$

with $\bar{u}_{n}^{s}(\mathbf{r}), \bar{u}_{s}^{s}(\mathbf{r})$ and $\bar{u}_{n}^{f}(\mathbf{r})$ respectively the prescribed values of the displacement components of the solid phase in the normal and tangential direction to the boundary and the prescribed value of the displacement of the fluid phase in the normal direction to the boundary.

- mechanical boundary conditions, the stress resultants are prescribed:

$$
\mathbf{r} \in \Gamma_{m e}:\left\{\begin{array}{l}
R_{\sigma_{n}^{s}}(\mathbf{r})=\sigma_{n}^{s}(\mathbf{r})-\bar{\sigma}_{n}^{s}(\mathbf{r})=0 \\
R_{\sigma_{s}^{s}}(\mathbf{r})=\sigma_{s}^{s}(\mathbf{r})-\bar{\sigma}_{s}^{s}(\mathbf{r})=0 \\
R_{\sigma^{f}}(\mathbf{r})=\sigma_{n}^{f}(\mathbf{r})-\bar{\sigma}^{f}(\mathbf{r})=0
\end{array}\right.
$$

with $\bar{\sigma}_{n}^{s}(\mathbf{r}), \bar{\sigma}_{s}^{s}(\mathbf{r})$ and $\bar{\sigma}^{f}(\mathbf{r})$ respectively the prescribed values of the normal and tangential stress resultant components of the solid phase in the normal and tangential direction to the boundary and the prescribed hydrostatic stress of the fluid phase.

- mixed boundary conditions:

$$
\mathbf{r} \in \Gamma_{m i}:\left\{\begin{array}{l}
R_{u_{n}^{s}}(\mathbf{r})=u_{n}^{s}(\mathbf{r})-\bar{u}_{n}^{s}(\mathbf{r})=0 \\
R_{u_{n}^{f}}(\mathbf{r})=u_{n}^{f}(\mathbf{r})-\bar{u}_{n}^{f}(\mathbf{r})=0 \\
R_{\sigma_{s}^{s}}(\mathbf{r})=\sigma_{s}^{s}(\mathbf{r})-\bar{\sigma}_{s}^{s}(\mathbf{r})=0
\end{array}\right.
$$

For a sliding edge, the prescribed values of $\bar{u}_{n}^{s}(\mathbf{r}), \bar{u}_{n}^{f}(\mathbf{r})$ and $\bar{\sigma}_{s}^{s}(\mathbf{r})$ are zero.

\subsection{Acoustic-poroelastic interactions}

On the interface $\Gamma_{C}$ in between the acoustic and the poroelastic subdomain the four following coupling conditions are imposed:

$$
\mathbf{r} \in \Gamma_{C}:\left\{\begin{array}{l}
R_{\sigma_{n, C}^{s}}(\mathbf{r})=\sigma_{n}^{s}(\mathbf{r})+(1-h) p(\mathbf{r})=0 \\
R_{\sigma_{n, C}^{f}}(\mathbf{r})=\sigma_{n}^{f}(\mathbf{r})+h p(\mathbf{r})=0 \\
R_{\sigma_{s, C}^{s}}(\mathbf{r})=\sigma_{s}^{s}(\mathbf{r})=0 \\
R_{u, C}(\mathbf{r})=\mathcal{L}_{v}(p(\mathbf{r}))-j \omega\left((1-h) u_{n}^{s}(\mathbf{r})+h u^{f}(\mathbf{r})\right)=0
\end{array}\right.
$$

$h$ is the porosity of the poroelastic material, defined as the ratio of the volume of fluid phase and the total volume of porous material. The first condition requires that the normal stress acting on 
the solid phase of the porous material is equal to $-(1-h)$ times the pressure in the acoustic field. The second equation gives a similar relation for the stress acting on the fluid phase. Since the air in the acoustic cavity is considered to be inviscous, the shear stress in the solid phase of the poroelastic material has to be zero. The final condition represents the continuity of the normal volume velocity. The first three boundary conditions are imposed on the poroelastic domain, the last one on the acoustic domain.

\section{The Wave Based Method}

First, this section introduces the methodology of the WBM for a general 2D bounded Helmholtz problem. The general problem setting, the basic concept and the model properties are described. Afterwards the WBM is applied to acoustic and poroelastic problems.

\subsection{Methodology of the Wave Based Method for a general 2D bounded Helmholtz problem}

This paragraph shortly addresses interior Helmholtz problems. For a detailed description, also including unbounded Helmholtz problems, the reader is referred to literature [12, 23]. This section adopts the terminology of Van Genechten et al. [23].

\subsection{General problem setting}

Consider a general two-dimensional bounded steady-state dynamic problem, which may consist of different media (for instance combined acoustic-structural, acoustic-poroelastic problems,...). The total problem domain $\Omega$ can be divided into a number of subdomains, $\Omega_{l}\left(\Omega=\bigcup_{l} \Omega_{l}\right)$, each containing a different medium designated by $l$. The mathematical formulation of the physics in each subdomain $\Omega_{l}$ results in $N_{H}$ second-order Helmholtz equation(s):

$$
\nabla^{2} u_{j}(\mathbf{r})+k_{j}^{2} u_{j}(\mathbf{r})=\mathcal{F}_{j}(\mathbf{r}), \mathbf{r} \in \Omega_{l}, j=1, \ldots, N_{H}
$$

In this equation, $\nabla^{2}$ is the Laplacian operator, $k_{j}$ is the physical wave number of the $j^{\text {th }}$ Helmholtz equation, which is determined by the physical properties of the medium inside the problem domain $\Omega_{l}, l$ designates the type of medium inside and $\mathcal{F}_{j}(\mathbf{r})$ represents non-homogenous forcing terms. The boundary of the total interior problem $\Gamma_{b}$ can be divided into non-overlapping parts: $\Gamma_{b}=\bigcup_{i} \Gamma_{i}$, on which different boundary conditions can be imposed. The boundary conditions can be written in the general form:

$$
\mathcal{B}_{i}\left(u_{j}(\mathbf{r})\right)=\overline{\mathcal{B}}_{i}(\mathbf{r}), \mathbf{r} \in \Gamma_{i}
$$

with $\mathcal{B}_{i}(\bullet)$ a general boundary differential operator and $\overline{\mathcal{B}}_{i}(\mathbf{r})$ an imposed boundary field. The Helmholtz equation(s) (16) together with the applied boundary conditions (17) along $\Gamma_{b}$ defines a unique dynamic field $u_{j}(\mathbf{r})$ in the problem domain $\Omega$.

\subsection{Basic concept of the WBM}

The WBM [12] is a deterministic numerical method and belongs to the family of indirect Trefftz [13] approaches. Unlike the FEM, the WBM partitions the problem domain into a limited number of large convex subdomains. Convexity of the subdomains is a sufficient condition for the method to converge towards the exact solution of the problem [12]. Within each subdomain, the dynamic field variables are approximated using an set of wave functions which intrinsically satisfy the governing Helmholtz equation(s). The degrees of freedom (dofs) are the contribution factors of each wave 
function in this expansion. Enforcing the boundary and interface conditions along the subdomain boundaries using a Galerkin weighted residual formulation leads to a small, complex and frequency dependent system of equations which can be solved for the contribution factor of each wave function. The general modeling procedure of the WBM consists of the following 4 steps:

1. Partitioning of the considered problem domain into convex subdomains

2. Selection of a suitable set of wave functions for each subdomain

3. Construction of the WB system matrices via a weighted residual formulation of the boundary and interface conditions

4. Solution of the system of equations, yielding the wave function contribution factors and postprocessing of the dynamic variables

\subsubsection{Domain partitioning}

A sufficient condition for the theoretical convergence of the WBM is the convexity of the considered problem domain [12]. A non-convex domain $\Omega$ needs to be divided into $N_{\Omega}$ non-overlapping convex subdomains $\Omega=\bigcup_{\alpha=1}^{N_{\Omega}} \Omega^{(\alpha)}$. Obviously, if the problem domain $\Omega$ consists of different media $\Omega_{l}\left(\Omega=\bigcup_{l} \Omega_{l}\right)$, the partitioning has to be consistent. The internal boundaries in the domain $\Omega$ can be divided into two groups, respectively interfaces $\Gamma_{I}^{(\alpha, \beta)}$ between two subdomains containing the same material and interfaces $\Gamma_{C}^{(\alpha, \beta)}$ between subdomains containing different materials. Continuity conditions need to be imposed at the interfaces $\Gamma_{I}^{(\alpha, \beta)}$ and coupling conditions at the interfaces $\Gamma_{C}^{(\alpha, \beta)}$ between two adjacent subdomains $\Omega^{(\alpha)}$ and $\Omega^{(\beta)}$ :

$$
\begin{aligned}
& \mathcal{B}_{I}^{(\alpha, \beta)}\left(u_{j}^{(\alpha)}(\mathbf{r}), u_{j}^{(\beta)}(\mathbf{r})\right)=0, \mathbf{r} \in \Gamma_{I}^{(\alpha, \beta)} \\
& \mathcal{B}_{C}^{(\alpha, \beta)}\left(u_{j}^{(\alpha)}(\mathbf{r}), u_{j}^{(\beta)}(\mathbf{r})\right)=0, \mathbf{r} \in \Gamma_{C}^{(\alpha, \beta)}
\end{aligned}
$$

with $\mathcal{B}_{\star}^{(\alpha, \beta)}(\bullet, *)=\mathcal{B}_{\star}^{(\alpha)}(\bullet)+\mathcal{B}_{\star}^{(\beta)}(*)$ a general boundary differential operator expressing the continuity constraints on the fields $\bullet$ and $*$ and their derived quantities and $u_{j}^{(\alpha)}(\mathbf{r})$ and $u_{j}^{(\beta)}(\mathbf{r})$ the dynamic fields in both subdomains.

\subsubsection{Field variable expansions}

The steady-state dynamic field $(\mathrm{s}) u_{j}^{(\alpha)}(\mathbf{r})$ in the problem subdomain $\Omega^{(\alpha)}$ are approximated by a solution expansion $\hat{u}_{j}^{(\alpha)}(\mathbf{r})$ in terms of $n_{w}^{(\alpha)}$ wave functions $\Phi_{w}^{(\alpha)}$ :

$$
u_{j}^{(\alpha)}(\mathbf{r}) \simeq \hat{u}_{j}^{(\alpha)}(\mathbf{r})=\sum_{w=1}^{n_{w}^{(\alpha)}} u_{w}^{(\alpha)} \Phi_{w}^{(\alpha)}(\mathbf{r})+\hat{u}_{p, j}^{(\alpha)}(\mathbf{r})=\Phi_{\mathbf{w}}^{(\alpha)}(\mathbf{r}) \mathbf{u}_{\mathbf{w}}^{(\alpha)}+\hat{u}_{p, j}^{(\alpha)}(\mathbf{r}) .
$$

The wave function contribution factors $u_{w}^{(\alpha)}$ belonging to each of the wave functions are gathered in the vector of degrees of freedom $\mathbf{u}_{\mathbf{w}}{ }^{(\alpha)} . \hat{u}_{p, j}^{(\alpha)}$ represents a particular solution resulting from the combined source terms $\mathcal{F}_{j}(\mathbf{r})$ in the right hand side of the inhomogeneous Helmholtz equation (16). The row vector $\Phi_{\mathrm{w}}^{(\alpha)}$ collects the $n_{w}^{(\alpha)}$ wave functions $\Phi_{w}^{(\alpha)}$. Each wave function $\Phi_{w}^{(\alpha)}$ exactly satisfies the homogeneous part of the Helmholtz equation (16). For two-dimensional bounded domains, two sets of wave functions are distinguished, the $\mathrm{r}$ - and the s-set:

$$
\sum_{w=1}^{n_{w}^{(\alpha)}} u_{w}^{(\alpha)} \Phi_{w}^{(\alpha)}(\mathbf{r})=\sum_{w_{r}=1}^{n_{w_{r}}^{(\alpha)}} u_{w_{r}}^{(\alpha)} \Phi_{w_{r}}^{(\alpha)}(\mathbf{r})+\sum_{w_{s}=1}^{n_{w_{s}}^{(\alpha)}} u_{w_{s}}^{(\alpha)} \Phi_{w_{s}}^{(\alpha)}(\mathbf{r}),
$$


with $n_{w}^{(\alpha)}=n_{w_{r}}^{(\alpha)}+n_{w_{s}}^{(\alpha)}$. The wave functions are defined as:

$$
\Phi_{w}^{(\alpha)}(\mathbf{r}(x, y))=\left\{\begin{array}{c}
\Phi_{w_{r}}^{(\alpha)}(x, y)=\left\{\sin \left(k_{x w_{r}}^{(\alpha)} x\right), \cos \left(k_{x w_{r}}^{(\alpha)} x\right)\right\} e^{-j k_{y w_{r}}^{(\alpha)} y} \\
\Phi_{w_{s}}^{(\alpha)}(x, y)=e^{-j k_{x w_{s}}^{(\alpha)} x}\left\{\sin \left(k_{y w_{s}}^{(\alpha)} y\right), \cos \left(k_{y w_{s}}^{(\alpha)} y\right)\right\}
\end{array}\right.
$$

where $\{f(x, y), g(x, y)\} h(x, y)$ indicates the definition of two independent basis functions $f(x, y) . h(x, y)$ and $g(x, y) \cdot h(x, y)$. The only requirement for these wave functions to be exact solutions of the Helmholtz equation (16) is that the wave number components in (22) have to satisfy:

$$
\left(k_{x w_{r}}^{(\alpha)}\right)^{2}+\left(k_{y w_{r}}^{(\alpha)}\right)^{2}=\left(k_{x w_{s}}^{(\alpha)}\right)^{2}+\left(k_{y w_{s}}^{(\alpha)}\right)^{2}=k_{j}^{2}
$$

Desmet [12] proposes to select the following wave number components:

$$
\begin{aligned}
& \left(k_{x w_{r}}^{(\alpha)}, k_{y w_{r}}^{(\alpha)}\right)=\left(\frac{w_{1}^{(\alpha)} \pi}{L_{x}^{(\alpha)}}, \pm \sqrt{k_{j}^{2}-\left(k_{x w_{r}}^{(\alpha)}\right)^{2}}\right), \quad w_{1}^{(\alpha)}=0,1,2, \ldots \\
& \left(k_{x w_{s}}^{(\alpha)}, k_{y w_{s}}^{(\alpha)}\right)=\left( \pm \sqrt{k_{j}^{2}-\left(k_{y w_{s}}^{(\alpha)}\right)^{2}}, \frac{w_{2}^{(\alpha)} \pi}{L_{y}^{(\alpha)}}\right), \quad w_{2}^{(\alpha)}=0,1,2, \ldots
\end{aligned}
$$

The dimensions $L_{x}^{(\alpha)}$ and $L_{y}^{(\alpha)}$ are the dimensions of the (preferably smallest) bounding rectangle circumscribing the considered subdomain. Desmet [12] has shown that this set of wave functions forms a complete set and that the WBM will converge towards the exact solution of the problem given that the subdomains are convex. This infinite set of wave functions has to be truncated into a finite set in order to apply the WBM in a numerical scheme. The upper limit $w_{\bullet}^{(\alpha)}$ in the wave number selection (24)-(25) is chosen accordingly to the following truncation rule:

$$
\frac{w_{\bullet}^{(\alpha)} \pi}{L_{\bullet}^{(\alpha)}} \geqslant T \max _{j}\left(k_{j}\right) .
$$

The physical wavenumber $k_{j}$ corresponds to the largest physical wavenumber in the problem domain. In this way, the approximation fields in adjacent subdomains exhibit a similar spatial resolution along the interfaces. $T$ is a user defined truncation factor. The adopted truncation rule is frequency dependent and the number of included wave functions that have wave number components smaller than, or about equal to the truncation factor $T$ times the physical wavenumber $k_{j}$ are added to the wave function set. Pluymers [24] shows that this truncation rule leads to a balanced and approximately constant relative prediction error for all frequencies. Typical truncation factors are $T=1 \ldots 6$.

\subsubsection{Wave based model construction}

Within each subdomain, the proposed solution expansion (21) always exactly satisfies the Helmholtz equation(s) (16), irrespective of the values of the unknown contribution factors $u_{w}^{(\alpha)}$. These functions may however violate the imposed boundary and interface conditions. The errors on the boundaries and interfaces are minimized in an integral sense using a Galerkin weighted residual formulation. This yields an algebraic equation linking the unknown wave function contribution factors for subdomain $\Omega^{(\alpha)}$ to those of the adjacent subdomains. For each subdomain $\Omega^{(\alpha)}$, a similar 
algebraic equation can be constructed. The enforcement that these equations should hold for any combination of the weighting function results in a matrix system of equations:

$$
\left[\begin{array}{cccc}
A_{a a}^{(1,1)} & C_{a a}^{(1,2)} & \cdots & C_{a a}^{\left(1, N_{\Omega}\right)} \\
C_{a a}^{(2,1)} & A_{a a}^{(2,2)} & \cdots & C_{a a}^{\left(2, N_{\Omega}\right)} \\
\vdots & \vdots & \ddots & \vdots \\
C_{a a}^{\left(N_{\Omega}, 1\right)} & C_{a a}^{\left(N_{\Omega}, 2\right)} & \cdots & A_{a a}^{\left(N_{\Omega}, N_{\Omega}\right)}
\end{array}\right] \cdot\left[\begin{array}{c}
\mathrm{u}_{\mathrm{w}}^{(1)} \\
\mathbf{u}_{\mathrm{w}}^{(2)} \\
\vdots \\
\mathrm{u}_{\mathrm{w}}^{\left(\mathbf{N}_{\Omega}\right)}
\end{array}\right]=\left[\begin{array}{c}
\sum_{\beta} \mathrm{f}_{\mathrm{a}}^{(1, \beta)} \\
\sum_{\beta} \mathrm{f}_{\mathrm{a}}^{(2, \beta)} \\
\vdots \\
\sum_{\beta} \mathrm{f}_{\mathrm{a}}^{\left(\mathrm{N}_{\Omega}, \beta\right)}
\end{array}\right]
$$

The solution of these equations yields the contribution factor of each wave function in the solution expansion. For a full description of the variational theory, the system matrices $\mathbf{A}_{\mathbf{a}}^{(\bullet, \bullet \bullet}$, the coupling matrices $\mathbf{C}_{\mathbf{a a}}^{(\bullet \bullet \bullet)}$ and the loading vectors $\mathbf{f}_{\mathbf{a}}^{(\bullet)}$, the reader is referred to literature $[23,25]$.

\subsubsection{Solution of the system of equations and postprocessing}

The fourth step in the WB modeling process is the solution of the matrix equation (27). This yields the unknown contribution factor of each wave function. The back substitution of these values into the field variable expansions (20), yields an approximation $\hat{u}_{j}(\mathbf{r})$ of the dynamic field variables. Also higher-order variables can easily be obtained by applying the corresponding differential operator to the wave function expansion.

\subsection{Model properties}

This section shortly compares the WB modeling approach with the FEM and highlights the advantages and disadvantages.

\subsubsection{Problem discretization and degrees of freedom}

The dofs in a FE model are the nodal values whereas in the WB methodology, the unknown wave function contribution factors form the dofs. The FEM needs a discretization into a large number of elements to capture the dynamics of the field variables. A refinement of the mesh is necessary to retain the same accuracy at higher frequencies. Due to the fine discretization, the FEM has almost no restrictions regarding the geometric complexity. Since the WBM partitions the domain into large subdomains and uses exact solutions of the dynamic equations, a smaller number of dofs is sufficient to obtain a similar accuracy. Desmet [12] shows that a sufficient condition for the WB approximations to converge towards the exact solution is the convexity of the subdomain. This partitioning into convex domains is fixed for all frequencies. With increasing frequency, the number of wave functions is increased, see equation (26). Problem domains with a complex geometry require a large number of WB subdomains, negatively influencing the convergence rate of the method.

\subsubsection{Applicability of the method}

To increase the applicability of the method, two recent developments partially relax the severe constraints imposed by the convexity-requirement of the method. A hybrid FE-WBM approach [26] combines the ability of the FEM to handle the geometrically complex parts of the problem domain and the WBM to deal in a more efficient way with the large, convex parts of the problem domain. This already has been applied for purely acoustic and vibroacoustic problems. For some geometries, for instance a polygonal domain containing a number of inclusions, a partitioning into convex domains leads to a large number of subdomains or is even impossible. These limitations are alleviated by a so-called multi-level modeling framework $[23,27]$. The multiple objects in the 
problem domain are considered as different levels of the problem domain and each level considers the reflection and scattering of one object. These levels are linked together, yielding a single multilevel system, describing the entire problem domain. With these recent enhancements, the WBM is capable to deal with a wide range of dynamic problems.

\subsubsection{System matrix properties}

In general, the system matrices of the FEM are real, large, frequency independent and sparsely populated with a banded structure. These properties allow an efficient solution. Nevertheless, for some type of dynamic problems, like poroelastic materials, the material properties are complex and frequency dependent. In this case, the FE matrices have to be recalculated for each frequency and are complex, hampering the efficient solution and further limiting the practical frequency range. In contrast, the WB matrices are always complex, frequency dependent and fully populated. Hence complex and frequency dependent material properties do not adversely affect the properties of the system matrices.

\subsubsection{Accuracy of derived variables}

Since the FEM commonly applies polynomial shape functions to approximate the primary response variables, the higher order derived quantities are approximated less accurately. In the WBM, derivatives of wave functions are wave functions as well, with the same spatial resolution resulting in no additional loss in prediction accuracy.

\subsubsection{Computational performance}

The WBM, like any Trefftz based method, suffers from a poor numerical condition [28, 29]. This does however not prevent the practical convergence of the method but requires a high accuracy in constructing the WB matrices. Building the WB models involves the evaluation of highly oscillating integrals and is computationally more demanding than the construction of the FE matrices. The largest computational effort of the WBM is related to the evaluation of the system matrices [24]. In the past, several analytical and numerical integration techniques have been studied [30]. Numerical integration, applying the Gauss-Legendre integration rule was shown to be the most efficient for the kind of integrals to be solved for a WB scheme, since an efficient matrix multiplication [25] can be applied. This numerical integration technique is applied with a frequency-dependent number of quadrature points per oscillatory wavelength of the integrand. This results in an efficient evaluation of the system matrices. Nevertheless, because of the large number of FE degrees of freedom, the solution of the FE models takes much more time than the solution of the WB matrices. The convergence rate of the WBM is higher as compared to the FEM. This high convergence rate makes the WBM appropriate to tackle problems for an increased frequency range.

\subsection{WBM applied to acoustic problems}

In an acoustic subdomain the governing equation is a single Helmholtz equation (1) which corresponds to (16) with:

$$
\begin{array}{ll} 
& \text { - } N_{H}=1 \\
\text { - } & u_{1}(\mathbf{r})=p(\mathbf{r}), \\
\text { - } k_{1}=k_{f},
\end{array}
$$


- $\mathcal{F}_{1}(\mathbf{r})=-j \rho_{f} \omega q \delta\left(\mathbf{r}, \mathbf{r}_{\mathbf{q}}\right)$,

- $\Omega_{l}=\Omega_{a}$

Since the acoustic pressure inside the cavity is governed by a single Helmholtz equation, the total acoustic pressure field $p(\mathbf{r})$ in $\Omega_{a}$ can be approximated by applying the wave function selection as indicated in section 3.3.2. The particular solution term $\hat{p}_{p}^{(\alpha)}(\mathbf{r})$ for the non-homogeneous Helmholtz equation is given by:

$$
\hat{p}_{p}^{(\alpha)}(\mathbf{r})=\frac{\rho_{f} \omega Q^{(\alpha)}}{4} H_{0}^{(2)}\left(k d_{q}^{(\alpha)}\right) .
$$

with $d_{q}^{(\alpha)}$ the distance between the source and receiver position, $H_{0}^{(2)}(\bullet)$ the zero-order Hankel function of the second kind and $Q^{(\alpha)}=\int_{\Omega^{(\alpha)}} q^{(\alpha)} d \Omega$ the source strength.

In fact, to obtain convergence, the complete set (22) has to be used in the expansion set. For interior acoustic problems, however, the number of basis functions can be further optimized. Starting from the analytical solutions (22) of the Helmholtz equation, Desmet shows in [12] that the theoretical convergence of acoustic problems is retained if only the functions with a cosine as harmonic component are kept in the expansion set, provided that the considered domains are convex. This halves the number of basis functions and results in a substantial increase in efficiency.

Since the validation cases in this paper only consist of a convex problem domain, the enforcement of the inter-subdomain continuity conditions, for which Pluymers [24] proposes two types of formulations, are not discussed further in detail.

\subsection{WBM applied to poroelastic problems}

To apply the WB theory to poroelastic materials, the Biot equations have to be decoupled into a set of Helmholtz equations. As noted by Biot, poroelastic materials support three wave types simultaneously, one shear and two types of compressional waves. In the case that the material is isotropic, a possible decomposition for the solid displacements is given by:

$$
\left\{\begin{array}{l}
u_{x}^{s}(\mathbf{r}) \\
u_{y}^{s}(\mathbf{r})
\end{array}\right\}=\nabla\left(-\frac{1}{k_{l_{1}}^{2}} e_{1}^{s}(\mathbf{r})\right)-\frac{1}{k_{l_{2}}^{2}} e_{2}^{s}(\mathbf{r})+\nabla \times \frac{1}{k_{t}^{2}} \omega^{s}(\mathbf{r})
$$

with $e_{1}^{s}(\mathbf{r})$ and $e_{2}^{s}(\mathbf{r})$ two volumetric strains $\left(e^{s}(\mathbf{r})=e_{1}^{s}(\mathbf{r})+e_{2}^{s}(\mathbf{r})\right)$ and $\omega^{s}(\mathbf{r})$ the rotational strain of the solid phase. By substituting (29) in the Biot equations (10), one obtains the following set of three decoupled Helmholtz equations:

$$
\begin{aligned}
& \nabla^{4} e^{s}(\mathbf{r})+A_{1} \nabla^{2} e^{s}(\mathbf{r})+A_{2} e^{s}(\mathbf{r})=\left(\nabla^{2} e_{1}^{s}(\mathbf{r})+k_{l_{1}}^{2} e_{1}^{s}(\mathbf{r})\right)\left(\nabla^{2} e_{2}^{s}(\mathbf{r})+k_{l_{2}}^{2} e_{2}^{s}(\mathbf{r})\right)=0 \\
& \nabla^{2} \omega^{s}(\mathbf{r})+k_{t}^{2} \omega^{s}(\mathbf{r})=0
\end{aligned}
$$

Equation (30) indicates the existence of two compressional wave types with distinct wave numbers $k_{l_{1}}$ and $k_{l_{2}}$,

$$
\left\{\begin{array} { l } 
{ k _ { l _ { 1 } } = \sqrt { \frac { A _ { 1 } } { 2 } - \sqrt { \frac { A _ { 1 } ^ { 2 } } { 4 } - A _ { 2 } } } } \\
{ k _ { l _ { 2 } } = \sqrt { \frac { A _ { 1 } } { 2 } + \sqrt { \frac { A _ { 1 } ^ { 2 } } { 4 } - A _ { 2 } } } }
\end{array} \text { with } \left\{\begin{array}{l}
A_{1}=\omega^{2} \frac{\rho_{11}^{*} R-2 \rho_{12}^{*} Q+\rho_{22}^{*}\left(\lambda+2 N+\frac{Q^{2}}{R}\right)}{\left(\lambda+2 N+\frac{Q^{2}}{R^{2}}\right) R-Q^{2}} \\
A_{2}=\omega^{4} \frac{\rho_{11}^{*} \rho_{22}^{*}-\left(\rho_{12}^{*}\right)^{2}}{\left(\lambda+2 N+\frac{Q^{2}}{R}\right) R-Q^{2}}
\end{array}\right.\right.
$$


and equation (31) indicates the existence of one shear wave type with wave number $k_{t}$ :

$$
k_{t}=\omega \sqrt{\frac{\rho_{11}^{*} \rho_{22}^{*}-\left(\rho_{12}^{*}\right)^{2}}{N \rho_{22}^{*}}}
$$

The above discussion indicates that the mathematical description of a poroelastic problem results in a system of three Helmholtz equations (16) with:

- $N_{H}=3$

- $u_{1}(\mathbf{r})=e_{1}^{s}(\mathbf{r}), u_{2}(\mathbf{r})=e_{2}^{s}(\mathbf{r}), u_{3}(\mathbf{r})=\omega^{s}(\mathbf{r})$

- $k_{1}=k_{l_{1}}, k_{2}=k_{l_{1}}, k_{3}=k_{t}$

- $\mathcal{F}_{j}(\mathbf{r})=0$

- $\Omega_{l}=\Omega_{p e}$

According to the WB principles as explained in section 3, each of the three dynamic variables in the decoupled Biot equations, $e_{1}^{s}(\mathbf{r}), e_{2}^{s}(\mathbf{r})$ and $\omega^{s}(\mathbf{r})$, is approximated by a solution expansion of form (20). Since the three Helmholtz equations (30) are homogeneous, no particular solutions have to be defined. For the selection of the wave number components in the solution expansion of each dynamic variable, the corresponding wave numbers $k_{l_{1}}, k_{l_{2}}$ and $k_{t}$ are taken into account.

Each field variable $a$ can be written in function of the strain fields by applying a corresponding differential operator:

$$
a=\mathcal{L}_{a}\left[\begin{array}{c}
e_{1}^{s}(\mathbf{r}) \\
e_{2}^{s}(\mathbf{r}) \\
\omega^{s}(\mathbf{r})
\end{array}\right]
$$

The differential operators for the normal and tangential displacements and the stresses in both phases are defined as follows:

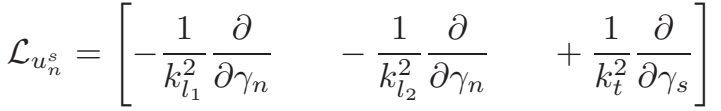

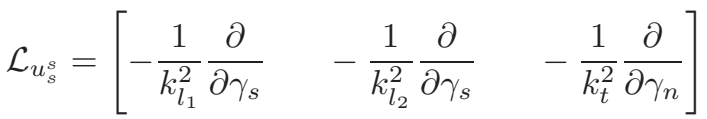

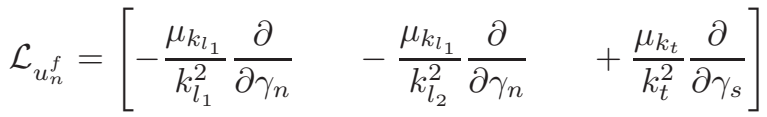

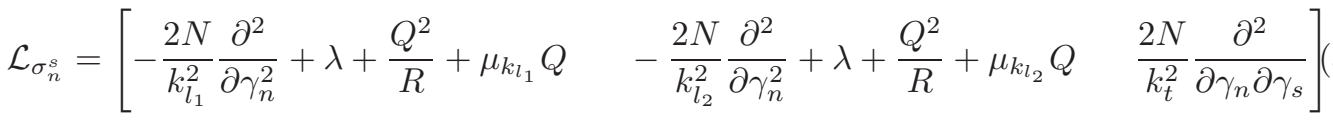

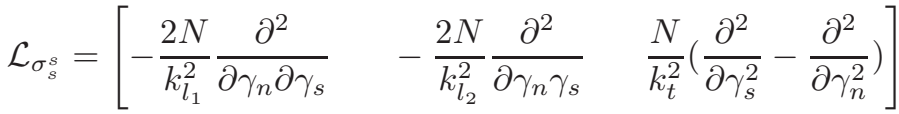

$$
\begin{aligned}
& \mathcal{L}_{\sigma^{f}}=\left[\begin{array}{lll}
Q+\mu_{k_{1}} R & Q+\mu_{k_{l_{2}}} R & 0
\end{array}\right]
\end{aligned}
$$

$\mu_{k_{l_{1}}}, \mu_{k_{l_{2}}}$ and $\mu_{k_{t}}$ are the ratios of the fluid and the solid displacements of the different wave types and are defined by Allard [2], see also appendix A. $\gamma_{\mathbf{s}}$ is the tangential direction of the boundary. 
The boundary residuals of the porous material can be determined using the same differential operators. The mutual coupling between the three wave field components is entirely contained within the boundary and continuity conditions specified along the boundaries and interfaces. For a full description of the WBM for uncoupled poroelastic materials, which also includes a decoupling of the Biot equations based on scalar and vector potentials and a description of the continuity conditions between two poroelastic subdomains, the reader is referred to [21].

\section{Stress singularities}

This paper focusses on the existence and treatment of stress singularities in poroelastic materials. As indicated by Sinclair [22], infinite values of stresses are physically impossible, but indicate that no finite stresses can be computed by the linear theory of elasticity. This is a consequence of three types of linearization which are made in the classical elasticity: the relationship between stresses and strains are linear, the strains depend linearly on the displacement gradients and the deflections are small. Singularities violate all three of these assumptions. Nevertheless they comply with all of the field equations. Once the equations are so simplified, compliance with the assumptions becomes unpoliced by the theory itself. Nevertheless, useful interpretations can be made if the analyst is aware of their presence and takes the singularities correctly into account [22]. Generally, two classes of stress singularities are distinguished. The first class originates from concentrated loads applied over regions with a vanishingly small area. This typically will lead to non-homogenous Helmholtz equations. In this case the definition of a particular solution is necessary, for example in the case for an acoustic point source see equation (28). Since the decoupling of the Biot equations leads to three homogenous Helmholtz equations, this type of singularities is not further considered for poroelastic materials. In the second class, singularities occur away from concentrated loads and always in concert with discontinuities. It is this class that will be considered in this paper. For a polygonal plate domain, discontinuity singularities can be expected at the corner points. Typically, this kind of singularity arises when the internal angle formed by the two sides of a corner exceeds a critical value, which depends on the imposed boundary conditions. As indicated by Vanmaele et al. [17], the WBM suffers from convergence problems when stress singularities are present. These problems arise from the global character of the wave functions, which have difficulties with capturing the singular behavior in the corner. As can be expected, the same kind of problems appear when stress singularities are present in corners of poroelastic problem domains. The objective of this section is to find analytical solutions that accurately describe the displacement and stress fields in the vicinity of corners with a singularity. In this way, a criterion can be defined to determine whether stress singularities are present. If so, the set of wave functions can be extended with additional enrichment functions which accurately represent the singular behavior close to the corner point.

To find an analytical solution that asymptotically approximates the displacement and stress fields in a corner of a poroelastic problem domain, some assumptions are made:

- The stresses in the vicinity of the corner point are hardly affected by the boundary conditions away from the considered corner point.

- Only homogeneous boundary conditions are considered, the imposed values of the prescribed stresses or displacements are zero.

- Only wedges consisting of a single poroelastic material are considered. 
Figure 2: 2D infinite poroelastic wedge domain

[31], polar cylindrical coordinates $r$ and $\theta$ are employed and the open angular region of interest $\mathcal{R}$ is given by:

$$
\mathcal{R}=\left\{(r, \theta) \mid 0<r<\infty, \quad-\frac{\alpha}{2}<\theta<\frac{\alpha}{2}\right\}
$$

The analytical solution needs to satisfy:

1. The Biot equations

2. The imposed boundary conditions

3. The regularity requirements at the vertex

Starting from the analytical solution for the strain fields, the stresses and their singular behavior can be evaluated.

\subsection{Analytical solution for an infinite wedge}

The solution for the displacement and the stress fields is defined starting from the decoupled Biot equations (30)-(31). Assuming a separable function for the strains $e_{1}^{s}(r, \theta), e_{2}^{s}(r, \theta)$ and $\omega^{s}(r, \theta)$ in polar coordinates leads to the following homogenous solution [32]:

$$
\begin{aligned}
e_{1}^{s}(\mathbf{r}) & =\sum_{b=1}^{\infty}\left[\cos \left(\lambda_{l_{1} b} \theta\right) R_{\lambda_{l_{1} b}}\left(k_{l_{1}} r\right)+\sin \left(\lambda_{l_{1} b}^{*} \theta\right) R_{\lambda_{l_{1} b}^{*}}\left(k_{l_{1}} r\right)\right] \\
e_{2}^{s}(\mathbf{r}) & =\sum_{b=1}^{\infty}\left[\cos \left(\lambda_{l_{2} b} \theta\right) R_{\lambda_{l_{2} b}}\left(k_{l_{2}} r\right)+\sin \left(\lambda_{l_{2} b}^{*} \theta\right) R_{\lambda_{l_{2} b}^{*}}\left(k_{l_{2}} r\right)\right] \\
\omega^{s}(\mathbf{r}) & =\sum_{b=1}^{\infty}\left[\cos \left(\lambda_{t b} \theta\right) R_{\lambda_{t b}}\left(k_{t} r\right)+\sin \left(\lambda_{t b}^{*} \theta\right) R_{\lambda_{t b}^{*}}\left(k_{t} r\right)\right]
\end{aligned}
$$


where

$$
R_{\lambda}(z)=A_{\lambda} J_{\lambda}(z)+B_{\lambda} Y_{\lambda}(z) .
$$

$A_{\lambda}$ and $B_{\lambda}$ are constants of integration and $J_{\lambda}(z)$ and $Y_{\lambda}(z)$ are respectively the ordinary and modified Bessel functions of the first kind. $\lambda_{\bullet}$ indicate the eigenvalues associated with symmetric eigenfunctions with respect to the axis $\theta=0$ and the eigenvalues $\lambda_{\bullet}^{*}$ are associated with antisymmetric eigenfunctions. This paper uses the following shortened notations:

$$
\begin{aligned}
\lambda_{l_{1}}=\lambda_{l_{1} b} & \text { or } \quad \lambda_{l_{1} b}^{*} \\
\lambda_{l_{2}}=\lambda_{l_{2} b} & \text { or } \quad \lambda_{l_{2} b}^{*} \\
\lambda_{t}=\lambda_{t b} & \text { or } \quad \lambda_{t b}^{*}
\end{aligned}
$$

depending on whether they are associated with symmetric or anti-symmetric eigenfunctions. The application of the boundary conditions at $\theta= \pm \alpha / 2$ leads to a system of equations which can be solved for the unknown integration constants:

$$
[\mathbf{A}]\{\mathbf{c}\}=\mathbf{0}
$$

with $[\mathbf{A}]$ the coefficient matrix and $\{\mathbf{c}\}$ the vector of unknown constants of integration. Since trivial solutions should be eliminated, the determinant of the coefficient matrix must be zero:

$$
\operatorname{det}[\mathbf{A}]=0
$$

and the eigenvalues $\lambda_{l_{1} b}, \lambda_{l_{1} b}^{*}, \lambda_{l_{2} b}, \lambda_{l_{2} b}^{*}, \lambda_{t b}, \lambda_{t b}^{*}$ are the solutions of this characteristic equation. Since it is assumed that the boundary conditions at infinity have no influence on the singular behavior at the corner, they are discarded. The absence of requirements at infinity renders solutions of (49) which are non-unique. Since the principle attribute of these fields is the characterization of all possible stress singularities at the corner point, this is not a problem [31]. In a configuration with a finite extent, locally containing the configuration studied above, the boundary conditions applied to the other boundaries will make the solution unique. In the WB approach, this is obtained by applying the weighted residual formulation.

At the corner point, the regularity requirements still have to be fulfilled. Consistent with [31], the following conditions have to be imposed at the tip of corner $(\mathrm{r}=0)$ :

$$
\begin{aligned}
& u_{r}^{s}(0, \theta)=\text { finite } \\
& u_{\theta}^{s}(0, \theta)=\text { finite } \\
& u_{r}^{f}(0, \theta)=\text { finite }
\end{aligned}
$$

Applying equation (29) in polar coordinates leads to:

$$
\begin{aligned}
& u_{r}^{s}(r, \theta)=-\frac{1}{k_{l_{1}}^{2}} \frac{\partial e_{1}^{s}}{\partial r}-\frac{1}{k_{l_{2}}^{2}} \frac{\partial e_{2}^{s}}{\partial r}+\frac{1}{k_{t}^{2} r} \frac{\partial \omega^{s}}{\partial \theta} \\
& u_{r}^{f}(r, \theta)=-\frac{\mu_{k_{l_{1}}}}{k_{l_{1}}^{2}} \frac{\partial e_{1}^{s}}{\partial r}-\frac{\mu_{k_{l_{2}}}}{k_{l_{2}}^{2}} \frac{\partial e_{2}^{s}}{\partial r}+\frac{\mu_{k_{t}}}{k_{t}^{2} r} \frac{\partial \omega^{s}}{\partial \theta} \\
& u_{\theta}^{s}(r, \theta)=-\frac{1}{k_{l_{1}}^{2}} \frac{1}{r} \frac{\partial e_{1}^{s}}{\partial \theta}-\frac{1}{k_{l_{2}}^{2}} \frac{1}{r} \frac{\partial e_{2}^{s}}{\partial \theta}-\frac{1}{k_{t}^{2}} \frac{\partial \omega^{s}}{\partial r}
\end{aligned}
$$


These expressions have to remain finite when $r \rightarrow 0$. Since (54) and (55) depend on the same derivatives and only differ in some constant scaling factors, both are leading to the same conditions. Introduction of the homogenous solutions (42)-(44) in expressions (54) and (56) leads to the following conditions for the possible displacement fields:

$$
\begin{aligned}
\left.u_{r}^{s}(r, \theta)\right|_{r=0}=\lim _{r \rightarrow 0}[ & c_{1}(\theta)\left[A_{\lambda_{l_{1}}}\left(J_{\lambda_{l_{1}}-1}\left(k_{l_{1}} r\right)-J_{\lambda_{l_{1}}+1}\left(k_{l_{1}} r\right)\right)\right. \\
& \left.+B_{\lambda_{l_{1}}}\left(Y_{\lambda_{l_{1}}-1}\left(k_{l_{1}} r\right)-Y_{\lambda_{l_{1}}+1}\left(k_{l_{1}} r\right)\right)\right] \\
& +c_{2}(\theta)\left[A_{\lambda_{l_{2}}}\left(J_{\lambda_{l_{2}}-1}\left(k_{l_{2}} r\right)-J_{\lambda_{l_{2}}+1}\left(k_{l_{2}} r\right)\right)\right. \\
& \left.+B_{\lambda_{l_{2}}}\left(Y_{\lambda_{l_{2}}-1}\left(k_{l_{2}} r\right)-Y_{\lambda_{l_{2}}+1}\left(k_{l_{2}} r\right)\right)\right] \\
& \left.+\frac{c_{3}(\theta)}{r}\left[A_{\lambda_{t}} J_{\lambda_{t}}\left(k_{t} r\right)+B_{\lambda_{t}} Y_{\lambda_{t}^{*}}\left(k_{t} r\right)\right]\right]=\text { finite } \\
\left.u_{\theta}^{s}(r, \theta)\right|_{r=0}=\lim _{r \rightarrow 0}[ & \frac{c_{4}(\theta)}{r}\left[A_{\lambda_{l_{1}}} J_{\lambda_{l_{1}}}\left(k_{l_{1}} r\right)+B_{\lambda_{1}} Y_{\lambda_{l_{1}}^{*}}\left(k_{l_{1}} r\right)\right] \\
& +\frac{c_{5}(\theta)}{r}\left[A_{\lambda_{l_{2}}} J_{\lambda_{l_{2}}}\left(k_{l_{2}} r\right)+B_{\lambda_{2}} Y_{\lambda_{l_{2}}^{*}}\left(k_{l_{2}} r\right)\right] \\
& +c_{6}(\theta)\left[A_{\lambda_{l_{1}}}\left(J_{\lambda_{t}-1}\left(k_{t} r\right)-J_{\lambda_{t}+1}\left(k_{t} r\right)\right)\right. \\
& \left.+B_{\lambda_{t}}\left(Y_{\lambda_{t}-1}\left(k_{t} r\right)-Y_{\lambda_{t}+1}\left(k_{t} r\right)\right)\right]=\text { finite }
\end{aligned}
$$

with $c_{\bullet}(\theta)$ a function that only depends on coordinate $\theta$. Since

$$
\lim _{r \rightarrow 0} Y_{\lambda}(z)=-\infty
$$

all the integration constants $B_{\lambda}$ have to be zero to fulfill the regularity conditions. Since Bessel functions of the first kind can be expanded using a power series [33]:

$$
J_{\lambda}(z)=\sum_{k=0}^{\infty} \frac{(-1)^{k}}{k ! \Gamma(\lambda+k+1)} \frac{z^{2 k+\lambda}}{2}
$$

the remaining expressions stay finite if all the eigenvalues are larger than 1. Taking this into account, the eigenfunctions become:

$$
\begin{array}{rlrl}
R_{\lambda_{l_{1}}} & =A_{\lambda_{l_{1}}} J_{\lambda_{l_{1}}}\left(k_{l_{1}} r\right) & \lambda_{l_{1}}>1 \\
R_{\lambda_{l_{2}}} & =A_{\lambda_{l_{2}}} J_{\lambda_{l_{2}}}\left(k_{l_{2}} r\right) & \lambda_{l_{2}}>1 \\
R_{\lambda_{t}} & =A_{\lambda_{t}} J_{\lambda_{t}}\left(k_{t} r\right) & \lambda_{t} & >1
\end{array}
$$


yielding the following admissible displacement fields:

$$
\begin{aligned}
& u_{r}^{s}(r, \theta)=-\frac{1}{2 k_{l_{1}}}\left[A_{\lambda_{l_{1} b}} \cos \left(\lambda_{l_{1} b} \theta\right)\left(J_{\lambda_{l_{1} b}-1}\left(k_{l_{1}} r\right)-J_{\lambda_{l_{1} b}+1}\left(k_{l_{1}} r\right)\right)\right. \\
& \left.+A_{\lambda_{l_{1}^{*} b}} \sin \left(\lambda_{l_{1} b}^{*} \theta\right)\left(J_{\lambda_{l_{1}^{*} b}-1}\left(k_{l_{1}} r\right)-J_{\lambda_{l_{1} b}^{*}+1}\left(k_{l_{1}} r\right)\right)\right] \\
& -\frac{1}{2 k_{l_{2}}}\left[A_{\lambda_{l_{2} b}} \cos \left(\lambda_{l_{2} b} \theta\right)\left(J_{\lambda_{l_{2} b}-1}\left(k_{l_{2}} r\right)-J_{\lambda_{l_{2} b}+1}\left(k_{l_{2}} r\right)\right)\right. \\
& \left.+A_{\lambda_{l_{2}^{*} b}} \sin \left(\lambda_{l_{2} b}^{*} \theta\right)\left(J_{\lambda_{l_{2} b}^{*}-1}\left(k_{l_{2}} r\right)-J_{\lambda_{l_{2} b}^{*}+1}\left(k_{l_{2}} r\right)\right)\right] \\
& +\frac{1}{k_{t}^{2} r}\left[-A_{\lambda_{t b}} \lambda_{t b} \sin \left(\lambda_{t b} \theta\right) J_{\lambda_{t b}}\left(k_{t} r\right)+A_{\lambda_{t b}^{*}} \lambda_{t b}^{*} \sin \left(\lambda_{t b}^{*} \theta\right) J_{\lambda_{t b}^{*}}\left(k_{t} r\right)\right] \\
& u_{r}^{f}(r, \theta)=-\frac{\mu_{k_{l_{1}}}}{2 k_{l_{1}}}\left[A_{\lambda_{l_{1} b}} \cos \left(\lambda_{l_{1} b} \theta\right)\left(J_{\lambda_{l_{1} b}-1}\left(k_{l_{1}} r\right)-J_{\lambda_{l_{1} b}+1}\left(k_{l_{1}} r\right)\right)\right. \\
& \left.+A_{\lambda_{l_{1}^{*} b}} \sin \left(\lambda_{l_{1} b}^{*} \theta\right)\left(J_{\lambda_{l_{1}^{*} b}-1}\left(k_{l_{1}} r\right)-J_{\lambda_{l_{1} b}^{*}+1}\left(k_{l_{1}} r\right)\right)\right] \\
& -\frac{\mu_{k_{l_{2}}}}{2 k_{l_{2}}}\left[A_{\lambda_{l_{2} b}} \cos \left(\lambda_{l_{2} b} \theta\right)\left(J_{\lambda_{l_{2} b}-1}\left(k_{l_{2}} r\right)-J_{\lambda_{l_{2} b}+1}\left(k_{l_{2}} r\right)\right)\right. \\
& \left.+A_{\lambda_{l_{2}^{*} b}} \sin \left(\lambda_{l_{2} b}^{*} \theta\right)\left(J_{\lambda_{l_{2} b}^{*}-1}\left(k_{l_{2}} r\right)-J_{\lambda_{l_{2} b}^{*}+1}\left(k_{l_{2}} r\right)\right)\right] \\
& +\frac{\mu_{k_{t}}}{k_{t}^{2} r}\left[-A_{\lambda_{t b}} \lambda_{t b} \sin \left(\lambda_{t b} \theta\right) J_{\lambda_{t b}}\left(k_{t} r\right)+A_{\lambda_{t b}^{*}} \lambda_{t b}^{*} \cos \left(\lambda_{t b}^{*} \theta\right) J_{\lambda_{t b}^{*}}\left(k_{t} r\right)\right] \\
& u_{\theta}^{s}(r, \theta)=\frac{1}{k_{l_{1}}^{2} r}\left[A_{\lambda_{l_{1} b}} \lambda_{l_{1} b} \sin \left(\lambda_{l_{1} b} \theta\right) J_{\lambda_{l_{1} b}}\left(k_{l_{1}} r\right)-A_{\lambda_{l_{1} b}^{*}} \lambda_{l_{1} b}^{*} \cos \left(\lambda_{l_{1} b}^{*} \theta\right) J_{\lambda_{l_{1} b}^{*}}\left(k_{l_{1}} r\right)\right] \\
& +\frac{1}{k_{l_{2}}^{2} r}\left[A_{\lambda_{l_{2} b}} \lambda_{l_{2} b} \sin \left(\lambda_{l_{2} b} \theta\right) J_{\lambda_{l_{2} b}}\left(k_{l_{2}} r\right)-A_{\lambda_{l_{2} b}^{*}} \lambda_{t b}^{*} \cos \left(\lambda_{l_{2} b}^{*} \theta\right) J_{\lambda_{l_{2} b}^{*}}\left(k_{l_{2}} r\right)\right] \\
& -\frac{1}{2 k_{t}}\left[A_{\lambda_{t b}} \cos \left(\lambda_{t b} \theta\right)\left(J_{\lambda_{t b}-1}\left(k_{t} r\right)-J_{\lambda_{t b}+1}\left(k_{t b} r\right)\right)\right. \\
& \left.+A_{\lambda_{t^{*} b}} \sin \left(\lambda_{t^{*} b}^{*} \theta\right)\left(J_{\lambda_{t b}^{*}-1}\left(k_{t} r\right)-J_{\lambda_{t b}^{*}+1}\left(k_{t} r\right)\right)\right]
\end{aligned}
$$

with $\lambda_{l_{1}}, \lambda_{l_{2}}, \lambda_{t}>1$

\subsection{The strain and the stress field in the vicinity of the corner}

This paragraph investigates when stress singularities are present. Starting from the analytical solution for the displacement fields, admissible strain and stress fields can be determined in the vicinity of the corner. The strains can be written as:

$$
\begin{aligned}
e_{r}^{\bullet}(r, \theta) & =\frac{\partial u_{r}^{\bullet}(r, \theta)}{\partial r} \\
e_{\theta}^{\bullet}(r, \theta) & =\frac{1}{r} \frac{\partial u_{\theta}^{\bullet}(r, \theta)}{\partial \theta}+\frac{u_{r}^{\bullet}(r, \theta)}{r} \\
e_{r \theta}^{\bullet}(r, \theta) & =\frac{1}{r} \frac{\partial u_{r}^{\bullet}(r, \theta)}{\partial \theta}+\frac{\partial u_{\theta}^{\bullet}(r, \theta)}{\partial r}-\frac{u_{\theta}^{\bullet}(r, \theta)}{r}
\end{aligned}
$$


The stresses in both phases of the isotropic material are given by the constitutive relations (8) and can be expressed in polar coordinates:

$$
\begin{aligned}
\sigma_{r}^{s}(r, \theta) & =\left(\lambda+\frac{Q^{2}}{R}\right) e^{s}(r, \theta)+Q e^{f}(r, \theta)+2 N e_{r}^{s}(r, \theta) \\
\sigma_{\theta}^{s}(r, \theta) & =\left(\lambda+\frac{Q^{2}}{R}\right) e^{s}(r, \theta)+Q e^{f}(r, \theta)+2 N e_{\theta}^{s}(r, \theta) \\
\sigma_{r \theta}^{s}(r, \theta) & =N e_{r \theta}^{s}(r, \theta) \\
\sigma^{f}(r, \theta) & =Q e^{s}(r, \theta)+\operatorname{Re}^{f}(r, \theta)
\end{aligned}
$$

In this way, the possible stress distributions in the vicinity of the corner can be expressed in function of the admissible longitudinal and rotational strain fields (42)-(44), taking into account the eigenfunctions (61)-(63). Since the longitudinal strains $e_{1}^{s}(r, \theta)$ and $e_{2}^{s}(r, \theta)$ do not give rise to an unbounded value if $r \rightarrow 0$ the stresses in the fluid phase will always remain finite. However, singularities will exist in the solid phase if $e_{r}^{s}(r, \theta), e_{\theta}^{s}(r, \theta)$ or $e_{r \theta}^{s}(r, \theta)$ becomes singular when $r \rightarrow 0$. Substituting the expressions of the admissible displacement fields (64) and (66) in the expressions of the strains (67)-(69) and using the power series expansion for the Bessel function of the first kind (60), it can easily be verified when singularities can exist. For example the possible fields for the radial strain $e_{r}^{s}(r, \theta)$ are given by:

$$
\begin{aligned}
e_{r}^{s}(r, \theta)=-\frac{1}{4}[ & A_{\lambda_{l_{1} b}} \cos \left(\lambda_{l_{1} b} \theta\right)\left(J_{\lambda_{l_{1} b}-2}\left(k_{l_{1}} r\right)-2 J_{\lambda_{l_{1} b}}\left(k_{l_{1}} r\right)+J_{\lambda_{l_{1} b}+2}\left(k_{l_{1}} r\right)\right) \\
& \left.+A_{\lambda_{l_{1}^{*} b}} \sin \left(\lambda_{l_{1} b}^{*} \theta\right)\left(J_{\lambda_{l_{1}^{*} b}-2}\left(k_{l_{1}} r\right)-2 J_{\lambda_{l_{1}^{*} b}}\left(k_{l_{1}} r\right)+J_{\lambda_{l_{1} b}^{*}+2}\left(k_{l_{1}} r\right)\right)\right] \\
-\frac{1}{4}[ & A_{\lambda_{l_{2} b}} \cos \left(\lambda_{l_{2} b} \theta\right)\left(J_{\lambda_{l_{2} b}-2}\left(k_{l_{2}} r\right)-2 J_{\lambda_{l_{2} b}}\left(k_{l_{2}} r\right)+J_{\lambda_{l_{2} b}+2}\left(k_{l_{2}} r\right)\right) \\
& \left.+A_{\lambda_{l_{2}^{*} b} b} \sin \left(\lambda_{l_{2} b}^{*} \theta\right)\left(J_{\lambda_{l_{2} b}^{*}-2}\left(k_{l_{2}} r\right)-2 J_{\lambda_{l_{2} b}^{*}}\left(k_{l_{2}} r\right)+J_{\lambda_{l_{2} b}^{*}+2}\left(k_{l_{2}} r\right)\right)\right] \\
& -\frac{1}{k_{t}^{2} r^{2}}\left[-A_{\lambda_{t b}} \lambda_{t b} \sin \left(\lambda_{t b} \theta\right) J_{\lambda_{t b}}\left(k_{t} r\right)+A_{\lambda_{t b}^{*}} \lambda_{t b}^{*} \cos \left(\lambda_{t b}^{*} \theta\right) J_{\lambda_{t b}^{*}}\left(k_{t} r\right)\right] \\
+ & \frac{1}{2 k_{t} r}\left[-A_{\lambda_{t b}} \lambda_{t b} \sin \left(\lambda_{t b} \theta\right)\left(J_{\lambda_{t b}-1}\left(k_{t} r\right)-J_{\lambda_{t b}+1}\left(k_{t} r\right)\right)\right. \\
& \left.+A_{\lambda_{t b}^{*}} \lambda_{t b}^{*} \cos \left(\lambda_{t b}^{*} \theta\right)\left(J_{\lambda_{t b}^{*}-1}\left(k_{t} r\right)-J_{\lambda_{t b}^{*}+1}\left(k_{t} r\right)\right)\right]
\end{aligned}
$$

When substituting the Bessel functions by their series expansion it is clear that the stress field $\sigma_{r}^{s}(r, \theta)$ becomes singular in the corner point if one of the eigenvalues becomes smaller than 2 . The order of the singularity is $\lambda-2$. Singularities in the other stress components of the solid phase can be studied by writing down similar expressions for $e_{\theta}^{s}(r, \theta)$ and $e_{r \theta}^{s}(r, \theta)$. The order of these singularities is also $\lambda-2$.

\subsection{Analytical solution for the sliding edge infinite wedge}

This paragraph studies the specific case of an infinite wedge with imposed sliding edge conditions on both edges and searches for the analytical solution by determining the required eigenvalues. The boundary conditions are given in section 2.2 and expressed in polar coordinates, they can be written 
as:

$$
\begin{gathered}
u_{\theta}^{s}\left(r, \pm \frac{\alpha}{2}\right)=0, \\
u_{\theta}^{f}\left(r, \pm \frac{\alpha}{2}\right)=0, \\
\sigma_{r \theta}^{s}\left(r, \pm \frac{\alpha}{2}\right)=0 .
\end{gathered}
$$

These six boundary conditions are applied to the homogenous solution expansions. The solution of the resulting characteristic equation leads to the following set of eigenvalues:

$$
\begin{array}{lll}
\lambda_{l_{1} b}=\frac{2 b \pi}{\alpha} & \lambda_{l_{2} b}=\frac{2 b \pi}{\alpha} & \lambda_{t b}=(2 b-1) \frac{\pi}{\alpha} \\
\lambda_{l_{1} b}^{*}=(2 b-1) \frac{\pi}{\alpha} & \lambda_{l_{2} b}^{*}=(2 b-1) \frac{\pi}{\alpha} & \lambda_{t b}^{*}=\frac{2 b \pi}{\alpha}
\end{array}
$$

with $b=1,2, \ldots$. The homogenous solutions, using the eigenfunctions (61)-(63) and the above defined eigenvalues form analytical solutions of the infinite wedge domain since they fulfill the boundary conditions, the decoupled Biot equations and the regularity conditions. Stress singularities are present if at least one of the eigenvalues is smaller than 2. For a corner with two sliding edges, this is true if the angle $\alpha$ exceeds the critical value $\pi / 2$.

In case a singularity is present in the corner, the corresponding homogenous solutions with eigenvalues $<2$ will be added to the regular wave function sets. An important remark here is that these solutions do not form a complete set and as a consequence they can only be used together with the conventional set of wave functions. The only objective of these functions is to improve the convergence of the WBM. They are associated with a corner and added to the wave function set(s) of the subdomain(s) to which the corner belongs. Since they accurately represent the singularity in the vicinity of the considered corner, this addition will lead to better convergence properties as will be shown in a validation study.

\subsection{Other radial boundary conditions}

It can be easily verified that it is impossible to find an exact dynamic solution in case other radial boundary conditions are imposed. This is similar as compared to plate bending and plate membrane problems. Also, in the case of poroelastic materials the eigenvalues can not be deduced from the radial boundary conditions. Vanmaele [17] proposes to start from the analytical solutions for static problems. These solutions do not form exact solutions of the dynamic problem and can as such not be incorporated in the WBM. The static solutions are only used to define static eigenvalues from which the dynamic ones can be derived. They are chosen such that the singular behavior in the vicinity of the corner is asymptotically the same as for the static case. The special purpose enrichment functions will in this case not fulfill the dynamic boundary conditions, but the singularity will have the correct order. For poroelastic materials, static solutions of an infinite wedge to study singularities are not yet defined in literature. This topic will be dealt with in a subsequent paper, together with inhomogeneous boundary conditions.

\section{Validation example}

This section demonstrates the computational efficiency of the WBM as compared to the FEM in the case that stress singularities are present. Firstly, the presence of stress singularities is 
studied with two FE models, validating the criterion developed theoretically in the previous section and indicating the problems arising when no special purpose functions are included. Next, the singularity theory is incorporated in the WBM. The special purpose enrichment functions are discussed and the performance of the classical and enriched WB predictions are compared with FE results, both concerning accuracy and calculation time. All WB routines are implemented in Matlab R2007b. The FE predictions are obtained using Comsol 3.5a. The FE model uses a $\left(\mathbf{u}^{\mathbf{s}}, \mathbf{u}^{\mathbf{f}}\right)$-formulation and includes four degrees of freedom per node for the poroelastic domain. Cubic Lagrangian triangular finite elements are used. The FE models are solved using a direct UMFPACK solver. The mentioned calculation times include both the construction and the solution times since the models are frequency dependent. All calculations are performed on a $2.80 \mathrm{GHz}$ Intel dual core processor running a Windows Vista operation system.

\subsection{Existence of stress singularities and impact on WB accuracy}

To verify the existence of stress singularities, two different coupled acoustic-poroelastic problems are considered, based on the geometry shown in figure 3. Both problem geometries consist of a $2 \mathrm{D}$ rectangular acoustic subdomain $\Omega_{a}$, containing air, and a triangular poroelastic subdomain $\Omega_{p e}$ consisting of a polyurethane foam. For problem geometry 1 , the angle $\beta$ is chosen to be $\pi / 3$. For problem geometry $2, \beta$ is $\arctan (0.2 / 0.5) \approx 0.12 \pi$. The material properties of both subdomains are given in table 1. Figure 4 displays the three different wave numbers existing in the polyurethane foam as a function of frequency which gives an indication of the spectral content of each wave type. Boundaries $\Gamma_{1}, \Gamma_{3}$ and the right half side of $\Gamma_{2}$ are rigid boundaries, equation (2) with $\bar{v}_{n}=0$. On the left hand side of $\Gamma_{2}$ a normal velocity with amplitude $1 \mathrm{~m} / \mathrm{s}$ is imposed, exciting the acoustic cavity. On the coupling edge $\Gamma_{C}$, the coupling conditions (15) are imposed. The edges $\Gamma_{1}^{\prime}$ and $\Gamma_{2}^{\prime}$ of the poroelastic domain are sliding edges with imposed conditions (14).

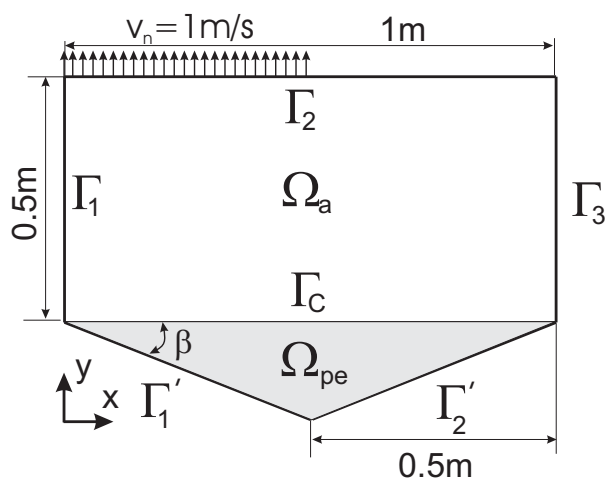

Figure 3: Problem geometry of a rectangular air cavity coupled with a triangular poroelastic domain 


\begin{tabular}{|c|c|}
\hline Air data & Porous material data \\
\hline \hline$k=2.57 \cdot 10^{-2} \mathrm{~W} /(\mathrm{mK})$ & $E_{s}=70 \cdot 10^{3} \mathrm{~Pa}$ \\
$c_{p}=1.005 \cdot 10^{3} \mathrm{~J} /(\mathrm{kgK})$ & $\eta_{l}=0.265$ \\
$R^{g a s}=286.7 \mathrm{~m}^{2} /\left(\mathrm{s}^{2} \mathrm{~K}\right)$ & $\nu=0.39$ \\
$T=293.15 \mathrm{~K}$ & $\rho_{s}=22.1 \mathrm{~kg} / \mathrm{m}^{3}$ \\
$\gamma=1.4$ & $\phi=0.98$ \\
$\nu_{f}=15.11 \cdot 10^{-6} \mathrm{~m}^{2} / \mathrm{s}$ & $\Lambda=1.1 \cdot 10^{-4}$ \\
$\rho_{f}=1.205 \mathrm{~kg} / \mathrm{m}^{3}$ & $\Lambda^{\prime}=7.42 \cdot 10^{-4}$ \\
& $\sigma_{\text {res }}=3.75 \cdot 10^{3} \mathrm{~kg} /\left(\mathrm{m}^{3} \mathrm{~s}\right)$ \\
& $\alpha_{\infty}=1.17$ \\
\hline
\end{tabular}

Table 1: Material data

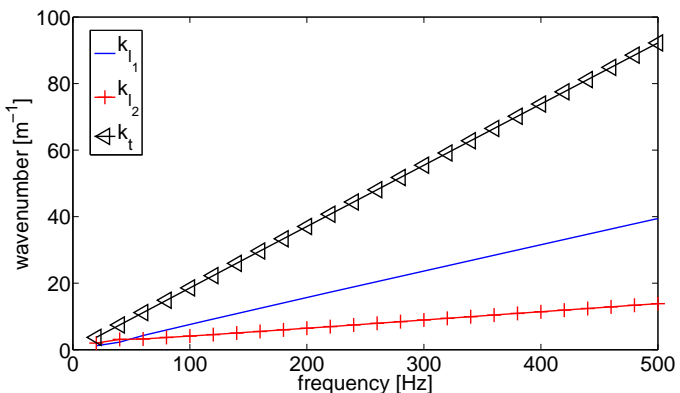

Figure 4: Wave numbers in the poroelastic domain in function of frequency

As predicted in section 4.3, stress singularities are present in a corner with imposed sliding edge conditions on both edges if the internal angle is larger than $\pi / 2$. Application of this theory to the problem geometries described above, predicts that the first problem configuration does not contain a singularity while the second one does. This is validated by means of two FE calculations at $200 \mathrm{~Hz}$. The FE model of problem geometry 1 contains 45785 dofs and the FE model of problem geometry 2 contains 53702 dofs. For both cases, five adaptive mesh refinements are performed, based on a $L_{2}$-norm of the error, to obtain accurate models. Figure 5 shows the obtained stress fields $\sigma_{x y}^{s}$ in the poroelastic subdomain for the first geometry. The stress values remain finite throughout the entire poroelastic domain.

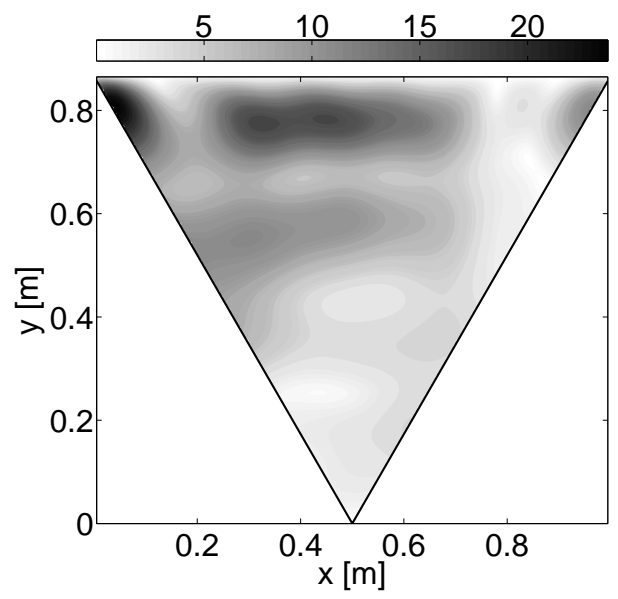

Figure 5: Predicted stress field $\sigma_{x y}^{s}\left[N / m^{2}\right]$ at $200 \mathrm{~Hz}$, calculated with the FEM

Figure 6 shows the stress field $\sigma_{x y}^{s}$ for the second case indicating a very steep gradient at the bottom of the poroelastic subdomain with a maximum of $\sim 3200 \mathrm{~N} / \mathrm{m}^{2}$ in the corner point, indicating that a stress singularity is present.

A WB model is constructed for both geometries to verify whether stress singularities have a negative influence on the accuracy of the WBM. For both models the same truncation factor, $T=2$, is applied to the wave type with the largest wave number and the number of wave functions of the other wave types are scaled such that each wave function expansion exhibits a similar spatial resolution. The FE models serve as a reference and are constructed based on 4 adaptive refinements 


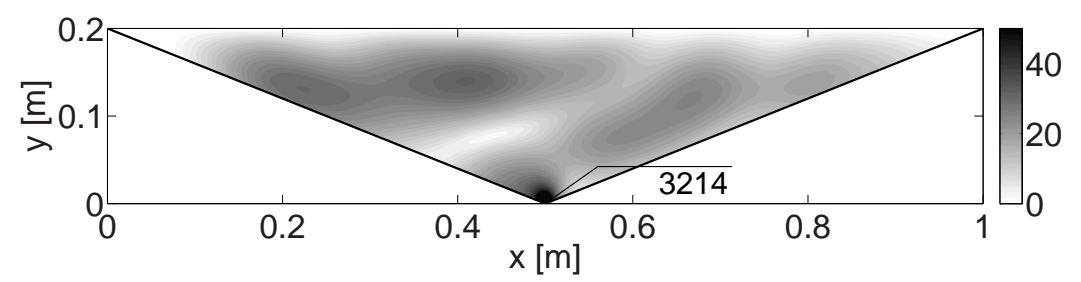

Figure 6: Predicted stress field $\sigma_{x y}^{s}\left[N / m^{2}\right]$ at $200 \mathrm{~Hz}$, calculated with the FEM

for each frequency. Figure 7 and figure 8 show the frequency response functions of $u_{y}^{s}$ in a point at the top left of the poroelastic domains. In the case without a singularity, the WBM is capable of predicting results as for the FEM. For the case with the singularity, even if the response point is located at a certain distance from the singular corner, the accuracy is clearly affected by the presence of the singularity.

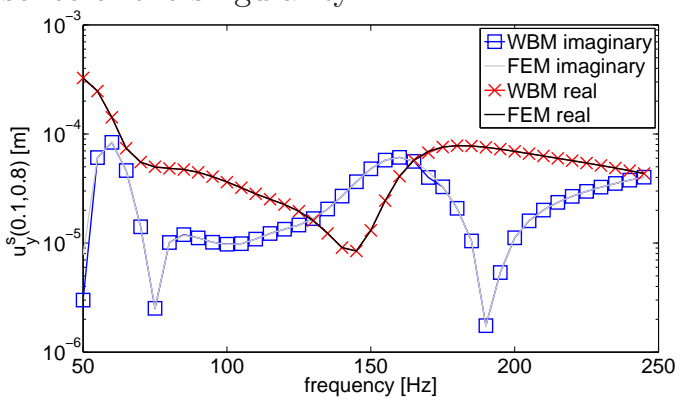

Figure 7: Frequency response function of $u_{y}^{s}(0.1,0.8)$ of the problem without a corner singularity

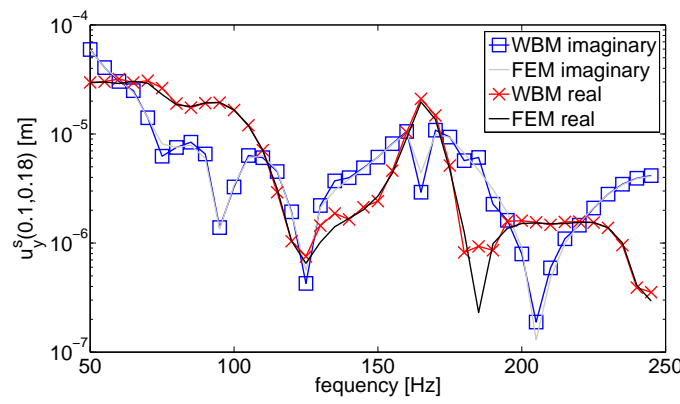

Figure 8: Frequency response function of $u_{y}^{s}(0.1,0.18)$ of the problem with a corner singularity

\subsection{Validation of the Wave Based Method with special purpose enrichment functions}

This paragraph focusses on the problem geometry 2 of figure 3 with $\beta=\arctan (0.2 / 0.5)$ which contains a corner singularity. It shows the beneficial effect of incorporating special purpose enrichment functions in the WBM. These functions will shortly be called 'corner functions' in this validation example and the use of the WBM with corner functions is indicated in graphs by WBM $\mathrm{CF}$. The WBM, the WBM CF and the FEM results are compared based on both accuracy and computational effort.

\subsubsection{WBM CF}

The eigenvalues of the characteristic equation (50) for the combination of two boundaries with imposed sliding edge conditions leads to eigenvalues given by the expressions (78)-(79). Singularities are present if $\lambda<2$. Only the eigenfunctions associated with eigenvalues $<2$ are added to the wave function set. Since the internal angle $\alpha$ is $136.4^{\circ}$, these eigenvalues are:

$$
\lambda_{l_{1}}^{*}=\lambda_{l_{2}}^{*}=\lambda_{t}=\frac{\pi}{\alpha}=1.320=\lambda_{C F}
$$


and the corresponding corner functions, added to the corresponding wave function sets for respectively $e_{1}^{s}(\mathbf{r}), e_{2}^{s}(\mathbf{r})$ and $\omega^{s}(\mathbf{r})$ are:

$$
\begin{aligned}
& \Phi_{l_{1}}^{C F}=\sin \left(\lambda_{C F} \theta\right) J_{\lambda_{C F}}\left(k_{l_{1}} r\right) \\
& \Phi_{l_{2}}^{C F}=\sin \left(\lambda_{C F} \theta\right) J_{\lambda_{C F}}\left(k_{l_{2}} r\right) \\
& \Phi_{t}^{C F}=\cos \left(\lambda_{C F} \theta\right) J_{\lambda_{C F}}\left(k_{t} r\right)
\end{aligned}
$$

Compared the WBM, the WBM CF has three more functions in its expansion sets.

\subsubsection{Comparison of $W B M$ and $W B M C F$}

A first indication of the difference in accuracy between the WBM and the WBM CF is given by a comparison of contour plots of the stress fields in the poroelastic domain. The calculations are performed at $200 \mathrm{~Hz}$ with a truncation factor of $T=1.5$ leading to 550 acoustic wave functions, 200 compressional wave functions for the strain expansion $e_{1}^{s}, 460$ compressional wave functions for the strain expansion $e_{2}^{s}$ and 84 shear wave functions. The WB CF model contains the same number of wave functions but is enriched with the three corner functions given above. Figure 9 compares the predicted stress fields $\sigma_{x y}^{s}(x, y)$ calculated with the WBM and the WBM CF. As expected, the stress field peaks at the bottom corner. In order to more clearly illustrate the adverse effect of the singularity on the WB predictions in the whole poroelastic field, twelve contour lines between $1 \mathrm{~N} / \mathrm{m}^{2}$ and $46 \mathrm{~N} / \mathrm{m}^{2}$ are added to the figures. These lines clearly show the presence of spurious oscillations in the WBM prediction which do not appear when adding the three corner functions. The values of the stress in the corner points are also given in the figures.

Figure 10 verifies the coupling condition between the stress $\sigma_{y}^{s}$ and the pressure in the acoustic domain at the interface for both the WBM and the WBM CF. These figures show that the coupling condition is much better fulfilled for the WBM CF. Large accuracy problems exist at the corners of the interface for results obtained with the WBM.

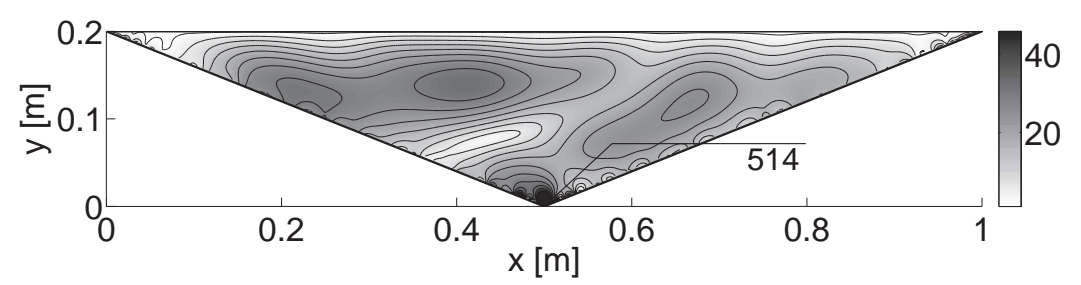

(a) WBM

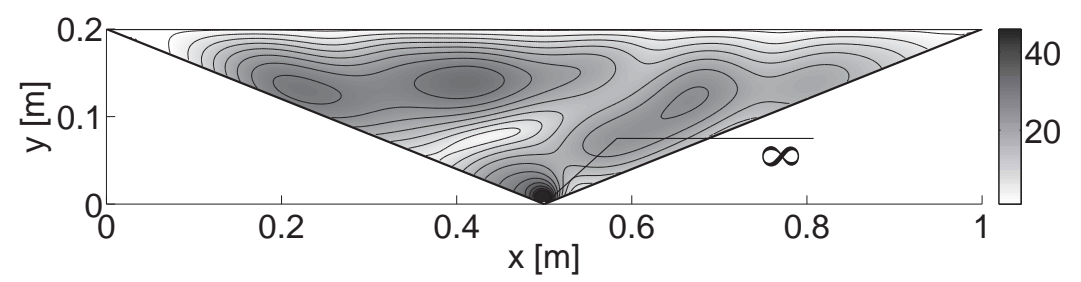

(b) WBM CF

Figure 9: Predicted stress field $\sigma_{x y}^{s}(x, y)\left[N / m^{2}\right]$ at $200 \mathrm{~Hz}$ 


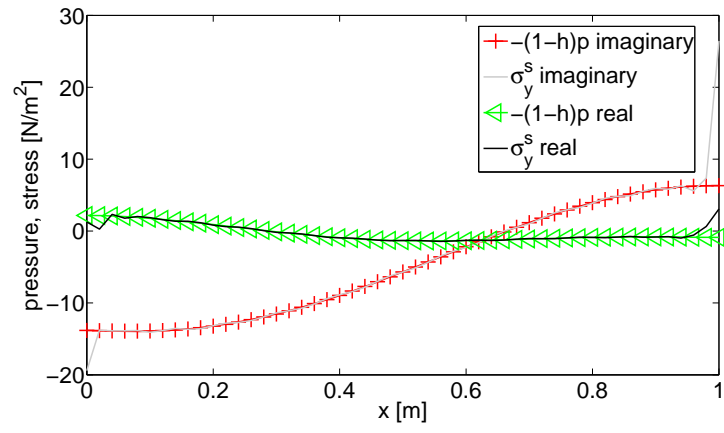

(a) WBM

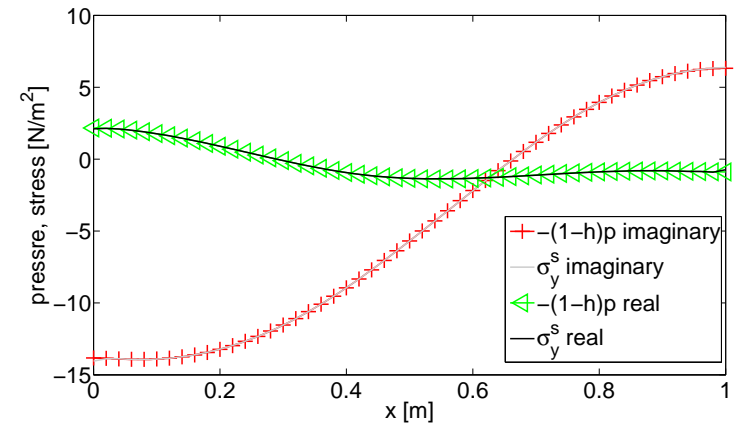

(b) WBM CF

Figure 10: $-(1-h) p$ and $\sigma_{y}^{s}$ calculated at $200 \mathrm{~Hz}$ along the coupling boundary

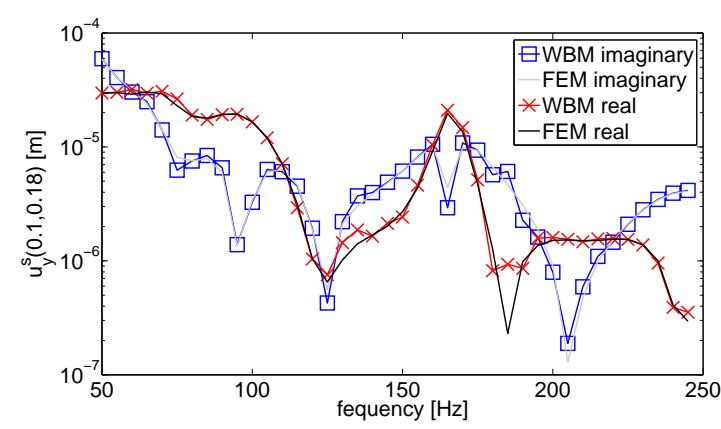

(a) WBM

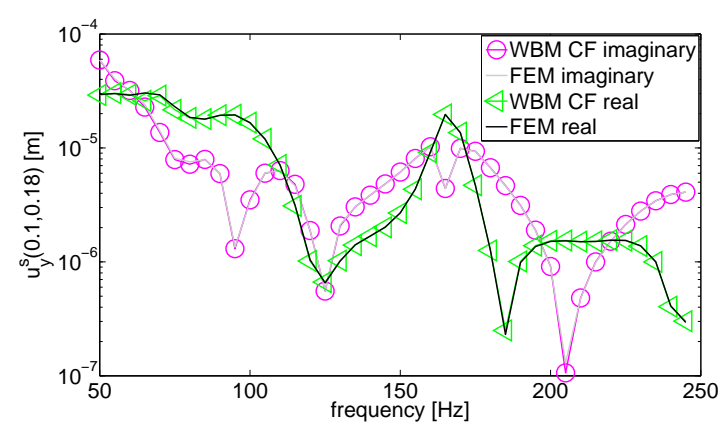

(b) WBM CF

Figure 11: Frequency response function of $u_{y}^{s}(0.1,0.18)$ calculated with the WBM, the WBM CF and the FEM

Figure 11 shows similar frequency response functions to those presented in the previous paragraph, calculated with both the WBM and the WBM CF and compared to the FE. These results confirm the increase in accuracy for the WBM CF.

\subsection{Comparison of computational efficiency}

Convergence curves at $200 \mathrm{~Hz}$ are calculated to demonstrate the beneficial convergence rate of the WBM CF as compared to both the WBM and the FEM. Nine Finite Element models are constructed and are summarized in table 2. One additional adaptive refinement is performed between each two subsequent FEM models. The finest FE model in this table serves as reference calculation. The calculation time $t_{t}$ is the total time needed to calculate the frequency dependent material properties, to construct and to solve the system matrices and to do the requested number of subsequent adaptive refinements. Different WB models are built with an increasing number of wave functions. Table 3 shows the minimum and the maximum number of wave functions of each type at $200 \mathrm{~Hz}$. 


\begin{tabular}{|c|c|c|c|}
\hline $\mathbf{n r}$ & $\sharp$ refs. & dofs & $\mathbf{t}_{t}[\mathbf{s}]$ \\
\hline 1 & 0 & 434 & 1.83 \\
2 & 1 & 2720 & 2.26 \\
3 & 2 & 8630 & 3.45 \\
4 & 3 & 23942 & 8.19 \\
5 & 4 & 53702 & 16.87 \\
6 & 5 & 121643 & 38.31 \\
7 & 6 & 261710 & 169.08 \\
8 & 7 & 560762 & 2538.35 \\
9 & 8 & 1071194 & - \\
\hline
\end{tabular}

\begin{tabular}{|c|c|c|c|c|}
\hline & $\mathbf{1}^{\text {st }}$ dilat. & $\mathbf{2}^{\text {nd }}$ dilat. & rot. & acoustic \\
\hline $\min$ & 12 & 28 & 4 & 34 \\
\hline $\max$ & 560 & 1280 & 236 & 1534 \\
\hline
\end{tabular}

Table 3: WBM model data at $200 \mathrm{~Hz}$

Table 2: FE model data at $200 \mathrm{~Hz}$

To compare the convergence rate of the different methods, the relative prediction error $\langle\epsilon\rangle$ of a variable $a$ is calculated as a function of CPU time. This prediction error is averaged over 26 response points which are equally distributed over the poro-elastic domain or the acoustic domain:

$$
\langle\epsilon\rangle=\frac{1}{26} \sum_{j=1}^{26} \epsilon_{j} \quad \text { with } \quad \epsilon_{j}=\frac{\left\|a\left(\mathbf{r}_{\mathbf{j}}\right)-a^{r e f}\left(\mathbf{r}_{\mathbf{j}}\right)\right\|}{\left\|a^{r e f}\left(\mathbf{r}_{\mathbf{j}}\right)\right\|}
$$

Figure 12 compares the convergence curves of the WBM, the WBM CF and the FEM at $200 \mathrm{~Hz}$ for five poroelastic variables and the acoustic pressure. The beneficial effect of adding corner functions to the wave function expansion is clearly illustrated. The accuracy increases and the convergence is stabilized. For higher accuracies, the WBM convergence curves can hardly keep up with those of the FEM, except for the acoustic pressure and the stress of the fluid phase, which are less affected by the singularity. The WBM CF convergence curves show a steep decline and stabilize at a fixed prediction accuracy. This type of convergence behavior typically indicates that the model which is used as a reference has not yet converged to a better accuracy than the model to which it is compared. The WBM CF outperforms the FEM in efficiency: a better accuracy is obtained at much less calculation time.

\section{Conclusions}

The WBM is a novel prediction technique which aims at relaxing the frequency limitations of element based techniques. Recently, the WBM has been applied to predict the dynamic behavior of poroelastic materials. This paper focuses on the existence of stress singularities, which can exist in the corners of poroelastic domains. Singularities are present if the corner angle exceeds a critical value, depending on the applied boundary conditions along the adjacent edges. If singularities are present, the accuracy of the WBM is clearly affected. This paper derives a general criterion to determine whether stress singularities are present and proposes to use special purpose enrichment functions in the wave function expansion set to accurately capture this singular behavior. These so-called corner functions are found by applying an asymptotic analysis to find solutions of the Biot equations which also fulfill the boundary conditions and regularity conditions at the corner tip. Only for the special case of sliding edge boundary conditions, exact analytical solutions can be found. For this type of singularities, the effect of adding corner functions to the wave function expansion is validated. A numerical example verifies the advantageous effects on both the accuracy and the convergence speed of the method. The performance of the WBM with corner functions is also compared to that of the FEM, indicating a higher convergence rate and the potential of the WBM for poroelastic calculations. 


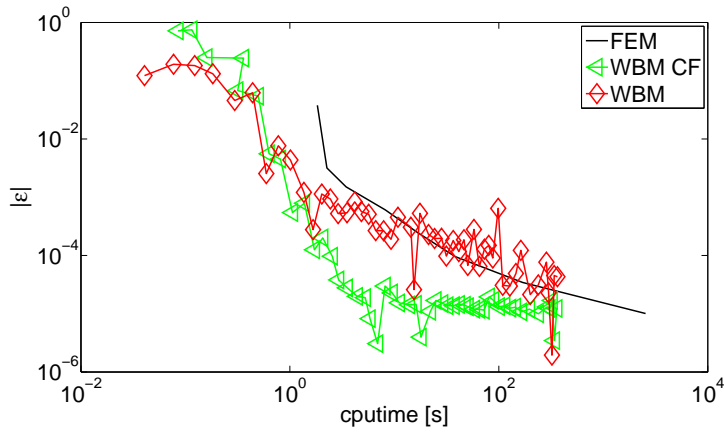

(a) $u_{x}^{s}(x, y)$

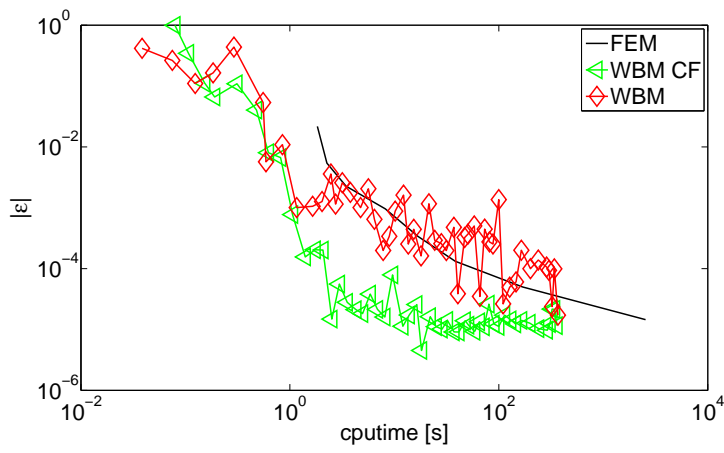

(c) $\sigma_{x}^{s}(x, y)$

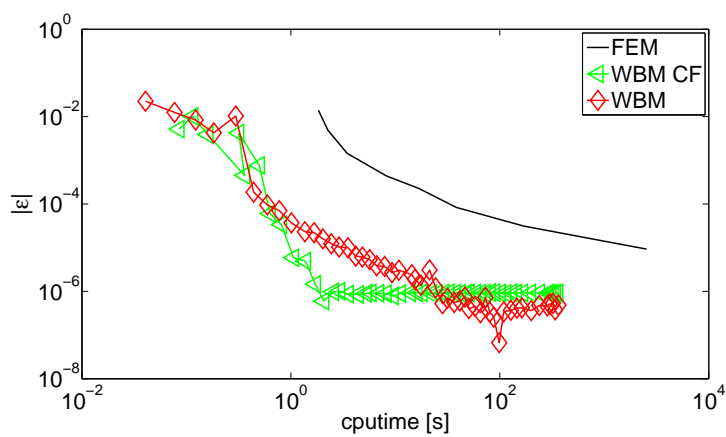

(e) $\sigma^{f}(x, y)$

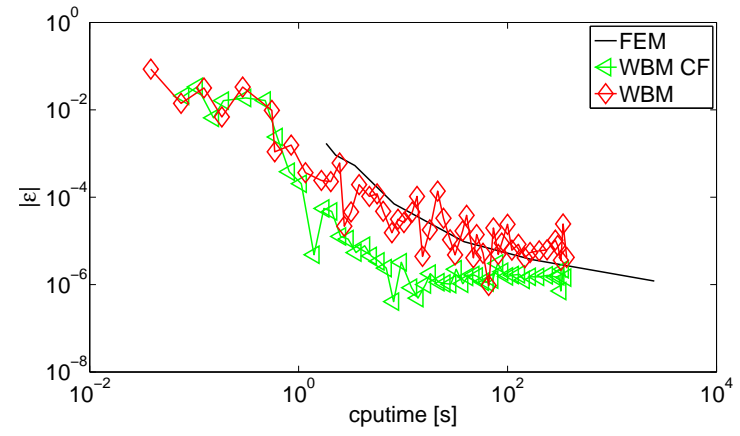

(b) $u_{y}^{f}(x, y)$

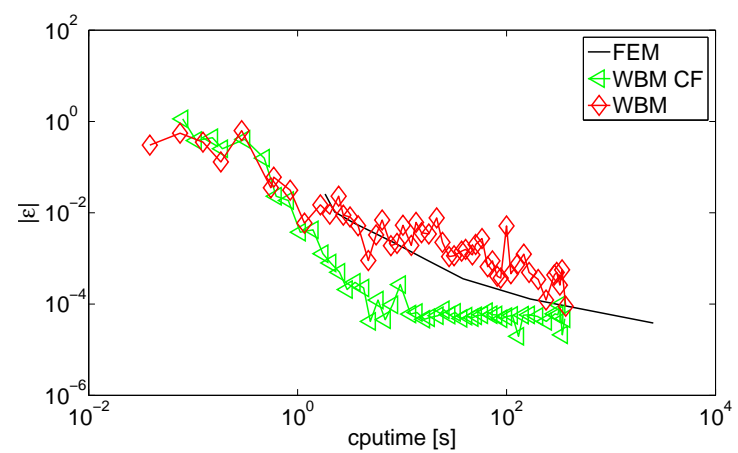

(d) $\sigma_{x y}^{s}(x, y)$

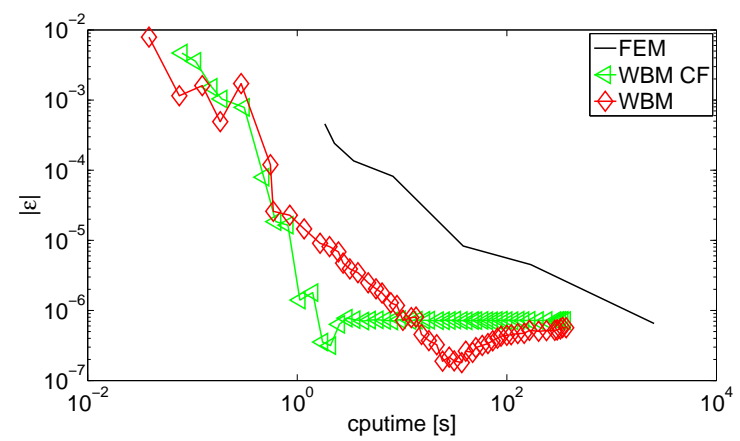

(f) $p(x, y)$

Figure 12: Convergence curves at $200 \mathrm{~Hz}$ for the WBM, the WBM CF and the FEM with FEM model number 9 as reference

\section{Aknowledgements}

Elke Deckers is a Doctoral Fellow of the Fund for Scientific Research-Flanders (F.W.O.), Belgium. Furthermore, the authors gratefully acknowledge the European Commission for their support of the ITN Marie Curie project GA-214909 "MIDFREQUENCY - CAE Methodologies for Mid-Frequency Analysis in Vibration and Acoustics 


\section{References}

[1] M. A. Biot, The theory of propagation of elastic waves in a fluid-saturated porous solid. I. Low frequency range. II. Higher frequency range, J. Acoust. Soc. Am. 28 (1956) 168-191.

[2] J.-F. Allard, Propagation of Sound in Porous Media: Modeling Sound Absorbing Materials, Elsevier, New York 1998.

[3] Y.J. Kang, J.S. Bolton, Finite element modeling of isotropic elastic porous materials coupled with acoustical finite elements, J. Acoust. Soc. Am. 98 (1995) 635-643.

[4] R. Panneton, N. Atalla, An efficient finite element scheme for solving the three dimensional poro-elasticity problems in acoustics, J. Acoust. Soc. Am. 101 (1997) 3287-3297.

[5] O. Dazel, B. Brouard, C. Depollier, S. Griffiths, An alternative Biot's displacement formulation for porous materials, J. Acoust. Soc. Am. 121 (2007) 3509-3516.

[6] N. Atalla, R. Panneton, P. Debergue, Enhanced weak integral formulation for the mixed ( $\underline{u}, \underline{p})$ poroelastic equations, J. Acoust. Soc. Am. 109 (2001) 3065-3068.

[7] P. Debergue, R. Panneton, N. Atalla, Boundary conditions for the weak formulation of the mixed (u,p) poroelasticity problem, J. Acoust. Soc. Am. 106 (1999) 2383-2390.

[8] N. Dauchez, S. Sahraoui, N. Atalla, Convergence of poroelastic finite elements based on Biot displacement formulation, J. Acoust. Soc. Am. 109 (2001) 33-40.

[9] N.-E. Hörlin, M. Nordström, P. Göransson, A 3-D hierarchical FE formulation of Biot's equations for elasto-acoustic modelling of porous media, J. Sound Vib. 245 (2001) 633-652.

[10] S. Rigobert, N. Atalla. F.C. Sgard, Investigation of the convergence of the mixed displacementpressure poroelastic materials using hierarchical elements, J. Acoust. Soc. Am. 114 (2003) 26072617.

[11] H. Kohno, K. J. Bathe, and J. C. Wright, A Finite Element Wave-Packet Procedure for Multiscale Wave Equations with Application to Plasma Waves, Comput. Struct. 88 (2010) 87- 94.

[12] W. Desmet, A Wave Based prediction technique for coupled vibro-acoustic analysis, K.U.Leuven, division PMA, PhD thesis 98D12, Leuven, 1998.

[13] E. Trefftz, Ein Gegenstück zum Ritzschen Verfahren, In: Proceedings of the 2nd International Congress on Applied Mechanics, Zürich, Switzerland, pp.131-137, 1926.

[14] B. Van Hal, W. Desmet, D. Vandepitte, A coupled Finite Element-Wave Based approach for the steady state dynamic analysis of acoustic systems, J. Comput. Acoust. 11 (2003) 285-303.

[15] B. Pluymers, B. Van Hal, D. Vandepitte, W. Desmet, Trefftz-based methods for time-harmonic acoustics, Arch. Comput. Methods Eng., 14 (2007) 343-381.

[16] C. Vanmaele, D. Vandepitte, W. Desmet, An efficient Wave Based prediction technique for plate bending vibrations, Comput. Methods Appl. Mech. Eng. 196 (2007) 3178-3189. 
[17] C. Vanmaele, D. Vandepitte, W. Desmet, An efficient Wave Based prediction technique for dynamic plate bending problems with corner stress singularities, Comput. Methods Appl. Mech. Eng. 198 (2009) 2227-2245.

[18] B. Pluymers, W. Desmet, D. Vandepitte, P. Sas, Application of an efficient Wave Based prediction technique for the analysis of vibro-acoustic radiation problems, J. Comput. Appl. Math. 168 (2004) 353-364.

[19] B. Pluymers, W. Desmet, D. Vandepitte, P. Sas, On the Use of a Wave Based Prediction Technique for Steady-State Structural-Acoustic Radiation Analysis, J. Comput. Model. Eng. \& Sci. 7 (2005) 173-184.

[20] E. Deckers, N.-E. Hörlin, D. Vandepitte, W. Desmet, A novel wave based prediction technique for the efficient dynamic modelling of poro-elastic materials, In: Proceedings of Euronoise, Edinburgh, Scotland, 2009.

[21] E. Deckers, N.-E. Hörlin, D. Vandepitte, W. Desmet, A novel wave based method for the efficient 2D dynamic modelling of the poro-elastic Biot equations, Submitted for publication in Comput. Methods Appl. Mech. Eng. (2010).

[22] G.B. Sinclair, Stress singularities in classical elasticity - I: Removal, interpretation and analysis, Applied Mechanics Reviews 57 (2004) 251-297.

[23] B. Van Genechten, K. Vergote, D. Vandepitte, W. Desmet, A multi-level wave based numerical modelling framework for the steady-state dynamic analysis of bounded Helmholtz problems with multiple inclusions, Comput. Methods Appl. Mech. Engrg. 199 (2010) 1881-1905.

[24] B. Pluymers, wave based modelling methods for steady-state vibro-acoustics, K.U.Leuven, division PMA, PhD. thesis 2006D04, Leuven, 2006.

[25] B. Van Hal, Auatomation and performance optimization of the wave based method for interior structural-acoustic problems, K.U.Leuven, division PMA, PhD. thesis 2004D07, 2004.

[26] B. Van Genechten, D. Vandepitte, W. Desmet, A direct hybrid Finite Element - Wave Based modelling technique for efficient mid-frequency coupled vibro-acoustic analysis, submitted to Comput. Methods Appl. Mech. Eng. (2010).

[27] B. Van Genechten, B. Bergen, D. Vandepitte, W. Desmet, A Trefftz-based numerical modelling framework for Helmholtz problems with complex multiple scatterer configurations. J. Comput. Physics, Accepted for publication, (2010).

[28] T. Huttunen, P. Gamallo, R.J. Astley, Comparison of two wave element methods for the Helmholtz problem, Commun. Numer. Methods Eng. 25 (2009) 35-52.

[29] A.P. Zielinski and I. Herrera, Trefftz method: fitting boundary conditions, Int. J. Numer. Methods Eng. 24 (1987) 871-891.

[30] E. Deckers, B. Drofmans, B. Van Genechten, B. Bergen, D. Vandepitte, W. Desmet, Splinebased boundaries: a first step towards generic geometic domain descriptions for efficient midfrequency acoustic analysis using the Wave Based Method, Accepted for publication with minor revisions in J. Comput. Appl. Math. (2010). 
[31] G.B. Sinclair, Stress singularities in classical elasticity - II: Asymptotic identification, Applied Mechanics Reviews 57 (2004) 385-439.

[32] P. Moon and D.E. Spencer, Field Theory Handbook Including Coordinate Systems, Differential Equations And Their Solutions, Springer-Verlag, 1961.

[33] M.D. Greenberg, Advanced Engineering Mathematics, second edition, Prentice Hall, New Jersey, 1998. 


\section{A. Biot theory parameters and nomenclature}

\begin{tabular}{|c|c|c|}
\hline & Description & Mathematical formula \\
\hline$b^{\prime}$ & Viscous drag coefficient & $b=\sigma_{r e s} h^{2} G_{J}(\omega)$ \\
\hline$B^{2}$ & Prandtl number & $B^{2}=\frac{c_{p} \mu_{f}}{k}$ \\
\hline$c$ & Speed of sound & $c=\sqrt{\gamma R^{k a s} T}$ \\
\hline$c_{p}$ & Specific heat & \\
\hline$e^{f}(\mathbf{r})$ & Volumetric strain in the fluid & $e^{f}(\mathbf{r})=\nabla \mathbf{u}^{\mathbf{f}}(\mathbf{r})$ \\
\hline$e^{s}(r)$ & Solid strain tensor & $\mathbf{e}^{\mathbf{s}}(\mathbf{r})=\frac{1}{2}\left(\nabla \mathbf{u}(\mathbf{r})+(\nabla \mathbf{u}(\mathbf{r}))^{T}\right)$ \\
\hline$e^{s}(\mathbf{r})$ & Volumetric strain in the solid & $e^{\frac{s}{s}}(\mathbf{r})=\nabla \mathbf{u}^{\mathbf{s}}(\mathbf{r})$ \\
\hline$e_{1}^{s}(\mathbf{r})$ & Volumetric strain according to $k_{l_{1}}$ & \\
\hline$\stackrel{e_{2}(\mathbf{r})}{E}$ & $\begin{array}{l}\text { Volumetric strain according to } k_{l_{2}} \\
\text { In-vacuo modulus of elasticity of the bulk solid phase }\end{array}$ & $E=E_{s}\left(1+j \eta_{l}\right)$ \\
\hline$G_{J}$ & Extra variable to take into account effective & $G_{J}=\sqrt{1+\frac{4 j \alpha_{\infty}^{2} \mu_{f} \rho_{f} \omega}{\sigma_{r e s}^{2} h^{2} \operatorname{Lambda}^{2}}}$ \\
\hline$h$ & $\begin{array}{c}\text { density and bulk modulus } \\
\text { Porosity }\end{array}$ & \\
\hline$k$ & conductivity of air & \\
\hline$k_{l_{1}}$ & Wave number of $1^{\text {st }}$ compressional wave type & equation (32) \\
\hline$k_{l_{2}}$ & Wave number of $2^{\text {nd }}$ compressional wave type & equation (32) \\
\hline$k_{t}^{2}$ & Wave number of shear wave type & equation (33) \\
\hline$K_{a}$ & Adiabatic Bulk modulus of the fluid & $K_{a}=\gamma R^{g a s} T \rho_{f}$ \\
\hline$K_{f}$ & Corrected Bulk modulus & $K_{f}=\frac{K_{a}}{\beta}$ \\
\hline$N$ & Shear modulus of the frame material & $N=\frac{E}{2(1+\nu)}$ \\
\hline$p(\mathbf{r})$ & Average fluid pore pressure & \\
\hline$Q$ & Compressional coupling factor & $Q=(1-h) K_{f}$ \\
\hline $\begin{array}{c}R \\
p^{g a s}\end{array}$ & Homogenized bulk modulus of the fluid phase & $R=h K_{f}$ \\
\hline $\begin{array}{l}R^{\text {gas }} \\
T\end{array}$ & $\begin{array}{c}\text { Gas constant of air } \\
\text { Temperature }\end{array}$ & \\
\hline$u^{f}(r)$ & Mean macroscopic fluid displacement vector & \\
\hline $\mathbf{u}^{\mathrm{s}}(\mathbf{r})$ & Mean macroscopic solid displacement vector & \\
\hline$\alpha_{\infty}$ & tortuosity & \\
\hline$\beta$ & Correction factor & $\beta=\gamma-\frac{\gamma-1}{1+\frac{8 \mu_{f}}{j \Lambda^{\prime 2} B^{2} \omega \rho_{f}} \sqrt{1+j \rho_{f} \frac{\omega B^{2} \Lambda^{\prime 2}}{16 \mu_{f}}}}$ \\
\hline$\eta_{l}$ & Loss factor & \\
\hline$\gamma$ & Ratio of specific heats & \\
\hline$\lambda$ & Static first Lamé coefficient of the frame material & $\lambda=\frac{E \nu}{(1+\nu)(1-2 \nu)}$ \\
\hline$\Lambda$ & Viscous characteristic length & \\
\hline$\Lambda^{\prime}$ & Thermal characteristic length & \\
\hline$\mu_{f}$ & Fluid dynamic viscosity & $\begin{array}{l}\mu_{f}=\rho_{f} \nu_{f} \\
\left(2 N+\lambda+Q^{2}\right) k_{l}^{2}-\omega^{2} \rho_{11}^{*}\end{array}$ \\
\hline$\mu_{k_{1}}$ & scaling constant & $\mu_{k_{l_{1}}}=\frac{\omega^{2} \rho_{12}^{*}-Q k_{l_{1}}^{2}}{\left(2 N+\lambda+Q^{2}\right) k_{l_{1}}^{2}-\omega^{2} \rho_{11}^{*}}$ \\
\hline$\mu_{k_{l_{2}}}$ & scaling constant & $\mu_{k_{l_{2}}}=\frac{\omega^{2} \rho_{12}^{*}-Q k_{l_{2}}^{2}}{l_{1}}$ \\
\hline$\mu_{k_{t}}$ & scaling constant & $\mu_{k_{t}}=-\frac{\rho_{12}^{*}}{\rho_{22}^{*}}$ \\
\hline$\nu$ & Poisson coefficient of the frame material & \\
\hline$\rho_{1}$ & Bulk frame mass density & $\rho_{1}=(1-h) \rho_{s}$ \\
\hline$\rho_{2}$ & Bulk fluid mass density & $\rho_{2}=h \rho_{s}$ \\
\hline$\rho_{11}^{*}$ & Effective frame mass density with viscous dissipation & $\rho_{11}^{*}=\rho_{1}+\rho_{a}+\frac{b}{j \omega}$ \\
\hline$\rho_{12}^{*}$ & Inertial mass coupling factor with viscous dissipation & $\rho_{12}^{*}=-\rho_{a}-\frac{b}{j \omega}$ \\
\hline$\rho_{22}^{*}$ & Effective fluid mass density with viscous dissipation & $\rho_{22}^{*}=\rho_{2}+\rho_{a}+\frac{b}{j \omega}$ \\
\hline$\rho_{a}$ & Inertial coupling term & $\rho_{a}=\rho_{f} \phi\left(\alpha_{\infty}-1\right)$ \\
\hline$\rho_{f}$ & Fluid density & \\
\hline $\begin{array}{c}\rho_{s} \\
\sigma^{f}(\mathbf{r})\end{array}$ & Mass density of the frame material & \\
\hline $\begin{array}{l}\sigma^{s}(\mathbf{r}) \\
\sigma^{s}(\mathbf{r})\end{array}$ & $\begin{array}{l}\text { Fluid stress tensor } \\
\text { Fluid stress tensor }\end{array}$ & \\
\hline$\sigma_{\text {res }}$ & $\begin{array}{l}\text { Fluid stress tensor } \\
\text { Static flow resistivity }\end{array}$ & \\
\hline$\Omega^{s}(\mathbf{r})$ & Rotational strain of the solid phase & $\Omega^{s}(\mathbf{r})=\nabla \times \mathbf{u}^{\mathbf{s}}(\mathbf{r})$ \\
\hline
\end{tabular}

Table 4: Notations used in the Biot equations 